\title{
Electronic absorption spectra of PAHs up to vacuum UV
}

\section{Towards a detailed model of interstellar PAH photophysics}

\author{
G. Malloci ${ }^{1,2}$, G. Mulas ${ }^{1,2}$, and C. Joblin ${ }^{3}$ \\ 1 INAF - Osservatorio Astronomico di Cagliari - Astrochemistry Group, Strada n.54, Loc. Poggio dei Pini, \\ 09012 Capoterra (CA), Italy \\ e-mail: [gmalloci;gmulas]@ca.astro.it \\ 2 Astrochemical Research in Space Network, http://www . ars-network.org \\ 3 Centre d'Étude Spatiale des Rayonnements, CNRS et Université Paul Sabatier, 9 avenue du colonel Roche, \\ 31028 Toulouse, France \\ e-mail: christine.joblin@cesr.fr
}

Received 29 March 2004 / Accepted 17 June 2004

\begin{abstract}
We computed the absolute photo-absorption cross-sections up to the vacuum ultaviolet (VUV) of a total of 20 Polycyclic Aromatic Hydrocarbons (PAHs) and their respective cations, ranging in size from naphthalene $\left(\mathrm{C}_{10} \mathrm{H}_{8}\right)$ to dicoronylene $\left(\mathrm{C}_{48} \mathrm{H}_{20}\right)$. We used an implementation in real time and real space of the Time-Dependent Density Functional Theory (TD-DFT), an approach which was proven to yield accurate results for conjugated molecules such as benzene. Concerning the low-lying excited states of $\pi^{*} \leftarrow \pi$ character occurring in the near-IR, visible and near-UV spectral range, the computed spectra are in good agreement with the available experimental data, predicting vertical excitation energies precise to within a few tenths of $\mathrm{eV}$, and the corresponding oscillator strengths to within experimental errors, which are indeed the typical accuracies currently achievable by TD-DFT. We find that PAH cations, like their parent molecules, display strong $\pi^{*} \leftarrow \pi$ electronic transitions in the UV, a piece of information which is particularly useful since a limited amount of laboratory data is available concerning the absorption properties of PAH ions in this wavelength range. Moreover, a detailed discussion of the UV-VUV properties of both neutral and cation species is presented. Concerning neutrals, the agreement with existing laboratory data is very good, the specific TD-DFT implementation used in this work apparently being able to reproduce the overall far-UV behaviour, including the broad absorption peak dominated by $\sigma^{*} \leftarrow \sigma$ transitions, which matches well both in position and width. The implications of these results are discussed in conjunction with the contribution PAH-like molecules are expected to give to the interstellar extinction curve.
\end{abstract}

Key words. astrochemistry - molecular data - molecular processes - ISM: molecules - ultraviolet: ISM - methods: numerical

\section{Introduction}

Based on their spectral properties, their high photo-stability and the fact that they are carbon-based, free gas-phase polycyclic aromatic hydrocarbons (PAHs) in different charge and hydrogenation states are commonly thought to be an important component of the interstellar medium (ISM). The original hypothesis appeared about twenty years ago (Léger \& Puget 1984; Allamandola et al. 1985), when these molecules were suggested as the most natural interpretation for the so called "Aromatic Infrared Bands" (AIBs), a set of discrete emission bands observed near 3.3, 6.2, 7.7, 8.6, 11.3 and $12.7 \mu \mathrm{m}$ in many dusty environments excited by UV photons (Léger et al. 1989; Allamandola et al. 1989). The AIBs are the spectral fingerprint of the excitation of vibrations in aromatic $\mathrm{C}-\mathrm{C}$ and C-H bonds (Duley \& Williams 1981). In the framework of the PAH model, following the absorption of a single UV photon via an electronic transition, a typical PAH quickly converts most or all of the excitation energy to vibrational energy in a low-lying electronic state (which most times is actually the ground state), being transiently heated to high temperatures (of the order of $10^{3} \mathrm{~K}$ ) due to its limited heat capacity (Andriesse 1978; Sellgren 1984); in a collision-free environment, it subsequently relaxes, emitting in its IR-active vibrational modes. Although several open questions remain, in the last two decades this "PAH hypothesis" has motivated many observational, experimental and theoretical efforts, which led to the speculation that these molecules and their derivatives could also give rise to other unidentified spectral features of interstellar origin: since large neutral PAHs and all ionised PAHs absorb in the visible, they were proposed to account for a subset of the Diffuse Interstellar Bands (DIBs) (Léger \& d'Hendecourt 1985; van der Zwet \& Allamandola 1985; Crawford et al. 1985). DIBs represent more than 
$\sim 300$ absorption features observed in the near-UV, visible and near-IR ranges along interstellar sigthlines reddened by interstellar dust (see e.g., Ehrenfreund \& Charnley 2000). It was also shown that mixtures of gaseous PAHs (present as ions, radicals as well as neutral species) could give an important contribution to the $2175 \AA$ bump and to the far-UV rise of the interstellar extinction curve (Joblin 1992; Joblin et al. 1992; Li \& Greenberg 1997). PAHs in the condensed form are known to be efficient emitters in the visible through fluorescence and phosphorescence mechanisms. Some data have also been recorded on isolated molecules in rare-gas matrices (see e.g., Joblin et al. 1999; Chillier et al. 2001) and on gas-phase species (Ossler et al. 2001a,b). For cations the data are more scarce, but perylene $\left(\mathrm{C}_{20} \mathrm{H}_{12}{ }^{+}\right)$was found to fluoresce in the visible and nearIR range (Joblin et al. 1995; Chillier et al. 2002). As originally suggested by d'Hendecourt et al. (1986), PAHs and their cations are therefore good candidates to account for the optical emission bands which appear to be related to some DIBs and are observed in peculiar interstellar objects (Schmidt et al. 1980; Scarrott et al. 1992; Kameswara-Rao \& Lambert 1993; Van Winckel et al. 2002).

Nowadays PAHs are seen as an intermediate stage between the gas and dust phases of the interstellar matter, i.e. large molecules or very small grains depending on the point of view, and, as such, they are included in some form in all interstellar dust models (see e.g., Li \& Greenberg 1997; Draine 2003). They are expected to exist in a wide variety of interstellar environments, in a complex statistical equilibrium of different charge and hydrogenation states (Bakes et al. 2001a,b; Le Page et al. 2001, 2003). PAHs are estimated to account for a substantial fraction of the total interstellar carbon budget (Boulanger 1999), and, absorbing stellar light, they are thought to play an important role in the energy balance of a galaxy: both by emitting via IR fluorescence about $15-30 \%$ of the total galactic emission (Boulanger 1999) and by coupling the stellar UV/visible photons to the ambient interstellar gas through photoelectric heating (Bakes \& Tielens 1994). The current state of our understanding of interstellar PAHs, the role they play in the physics and chemistry of the ISM and their spectroscopic properties, have been recently reviewed by Salama (1999).

In the light of the above discussion, the knowledge of the optical absorption properties of a large sample of PAHs in all their relevant ionisation and hydrogenation states is of fundamental importance for our understanding of the molecular mechanism which converts a large fraction of the interstellar radiation budget into the ubiquitous AIBs. While this concerns the whole energetic range of interstellar radiation up to $13.6 \mathrm{eV}$, very sparse data are available about the photoabsorption properties of neutral PAHs in the vacuum ultraviolet (VUV) at energies larger than about $7 \mathrm{eV}$ (Joblin 1992; Joblin et al. 1992; Gudipati et al. 1993, 1994, 1995; Robinson et al. 1997). For cations the situation is even worse (Salama 1999), due to the limitations which are intrinsic to the laboratory techniques more widely used (matrix isolation spectroscopy) until recently. Chillier et al. (1999) managed the first detection of an ultraviolet transition in an ionized $\mathrm{PAH}$, the perylene cation $\left(\mathrm{C}_{20} \mathrm{H}_{12}{ }^{+}\right)$. The above experimental result further supported the idea that PAHs, both neutral and cations, give an important contribution to the UV extinction curve. Yet, to the best of our knowledge, a detailed discussion of the UV-VUV properties of PAH ions has been missing until now.

On the contrary, a vast amount of information is available concerning the optical absorption properties of neutral and cationic PAHs in the near-IR, visible, near-UV spectral ranges, most obtained by matrix isolation spectroscopy studies (Salama \& Allamandola 1992; Salama et al. 1995; Salama 1999; Ruiterkamp et al. 2002), but including also some remarkable results in the gas-phase (Romanini et al. 1999; Pino et al. 1999; Biennier et al. 2003, 2004; Sukhorukov et al. 2004). The astrophysical implications of these data have been discussed in many papers in the literature, specifically with respect to the DIBs issue (Salama et al. 1996, 1999; Bréchignac \& Pino 1999).

Given the lack of accurate, quantitative laboratory experiments on the photo-absorption properties of a large number of PAHs and their ions up to the energy range excitable in a typical interstellar environment, the use of quantum-chemistry appears to be the next best alternative. Several papers making use of different quantum-chemical techniques, both semi-empirical (Parisel et al. 1992; Du et al. 1993; Negri \& Zgierski 1994) and ab initio (Negri \& Zgierski 1994; Niederalt et al. 1995; Hirata et al. 1999; Weisman et al. 2001, 2003; Hirata et al. 2003), to obtain the electronic excitation energies of PAH cations in the near IR to near UV range have appeared in the literature. Evidence for higher bright states in the UV in the spectra of PAHs ions has been given by Hirata et al. (1999), Weisman et al. (2001) and Weisman et al. (2003).

The main step forward achieved by the present paper with respect to previous theoretical studies lies in the spectral range covered: this is the first collection of individual photoabsorption cross-sections, computed in a uniform way from the visible to the VUV spectral domain and for a large sample of neutral and cationic PAHs.

In Sect. 2 we outline the computational approach that we used and validate it with available experimental data. In Sect. 3 we present the calculated spectra. In Sect. 4 we discuss the implications of the present results in the astrophysical context, mainly showing the relation beteween the contributions neutral and cationic PAHs are expected to give to the interstellar extinction curve. Our conclusions are presented in Sect. 5, where the impact these kind of studies may have for future spacebased single-molecule identifications is outlined.

\section{Computational approach}

\subsection{The TD-DFT method applied to PAHs}

The electronic absorption spectra of 20 different PAH species and their cations have been obtained in the framework of TimeDependent Density Functional Theory (TD-DFT) (Runge \& Gross 1984). This approach, the theory for electronic excitedstate properties corresponding to the traditional ground state DFT formulation, has been applied to a large number of systems in recent years (see e.g., Onida et al. 2002). TD-DFT calculations using different exchange-correlation functionals and basis sets are well known to be a powerful tool to calculate 
electronic excitation properties for neutral PAHs (Heinze et al. 2000; Parac \& Grimme 2003) as well as radical cations (Hirata et al. 1999) up to large species (Weisman et al. 2001, 2003). Halasinski et al. (2003) and Weisman et al. (2003) recently showed that interesting trends exist in the vertical excitation energies and oscillator strengths for homologous series of PAHs, a result which might have important implications for the longstanding DIBs mistery.

We used TD-DFT as implemented in the octopus computer program (Marques et al. 2003). In this code the timedependent Kohn-Sham equations are solved in real time and the wavefunctions are represented by their discretised values on a uniform spatial grid. We performed all calculations using the widely used local-density approximation, specifically with the exchange-correlation energy density of the homogeneous electron gas taken from Monte Carlo calculations (Ceperley \& Alder 1980) and then parametrized by Perdew \& Zunger (1981). Including gradient corrections in the exchangecorrelation functional yields only minor changes to the optical absorption spectrum predicted by the real-time real-space approach implemented in octopus (Marques et al. 2001). It is worth noting, in this respect, that the present application is rigorously ab initio from the point of view of the electronic degrees of freedom, while nuclei are considered to be classical point particles (Yabana \& Bertsch 1999). In addition, the ionic potentials are replaced by norm-conserving pseudo-potentials (Troullier \& Martins 1991). A thorough description of the program and the different numerical implementations it uses can be found in several papers of the authors (Marques et al. 2001; Castro et al. 2002; Marques et al. 2003) and in the dedicated web site http://www. tddft.org/programs/octopus.

The above method was proven to provide accurate photoabsorption spectra for conjugated molecules such as benzene (Yabana \& Bertsch 1999; Marques et al. 2003). We applied it to a sample of neutral PAHs, namely anthracene, pyrene, coronene and ovalene, and compared their computed spectra with the experimental absorption cross-sections measured in gas phase up to $\sim 30.0 \mathrm{eV}$ (Joblin 1992; Joblin et al. 2004). These latter data are extensively used in the astrophysical context (see e.g., Malloci et al. 2003; Le Page et al. 2003), being the only available experimental measurements of this kind. They were obtained at pressures of a few $10^{-2}$ torr and temperatures up to $\sim 700 \mathrm{~K}$ (depending on the vapor pressure of the molecule). In these experimental conditions the contributions of collisional and rotational broadenings were found to be negligible. At these high energies, the band width is important, due to the short lifetime of states. In the experimental curves, some (vibrational) sub-structure may also be unresolved due to the limited spectral resolution (see Joblin et al. 2004, for more details). The theoretical calculations we present here provide no information on this vibrational structure (cf. Sect. 2.2) seen.

We found a good agreement both for the lower-lying bands of $\pi^{*} \leftarrow \pi$ character, which was expected, and, more surprisingly, also for the collective single broad absorption peak at 17-18 eV, composed of $\sigma^{*} \leftarrow \sigma, \sigma^{*} \leftarrow \pi, \pi^{*} \leftarrow \sigma$ and Rydberg spectral transitions, with possible collective effects (e.g. plasmons, see Jochims et al. 1996, 1999). Figure 1 shows the calculated spectrum of neutral anthracene $\left(\mathrm{C}_{14} \mathrm{H}_{10}\right)$ and its

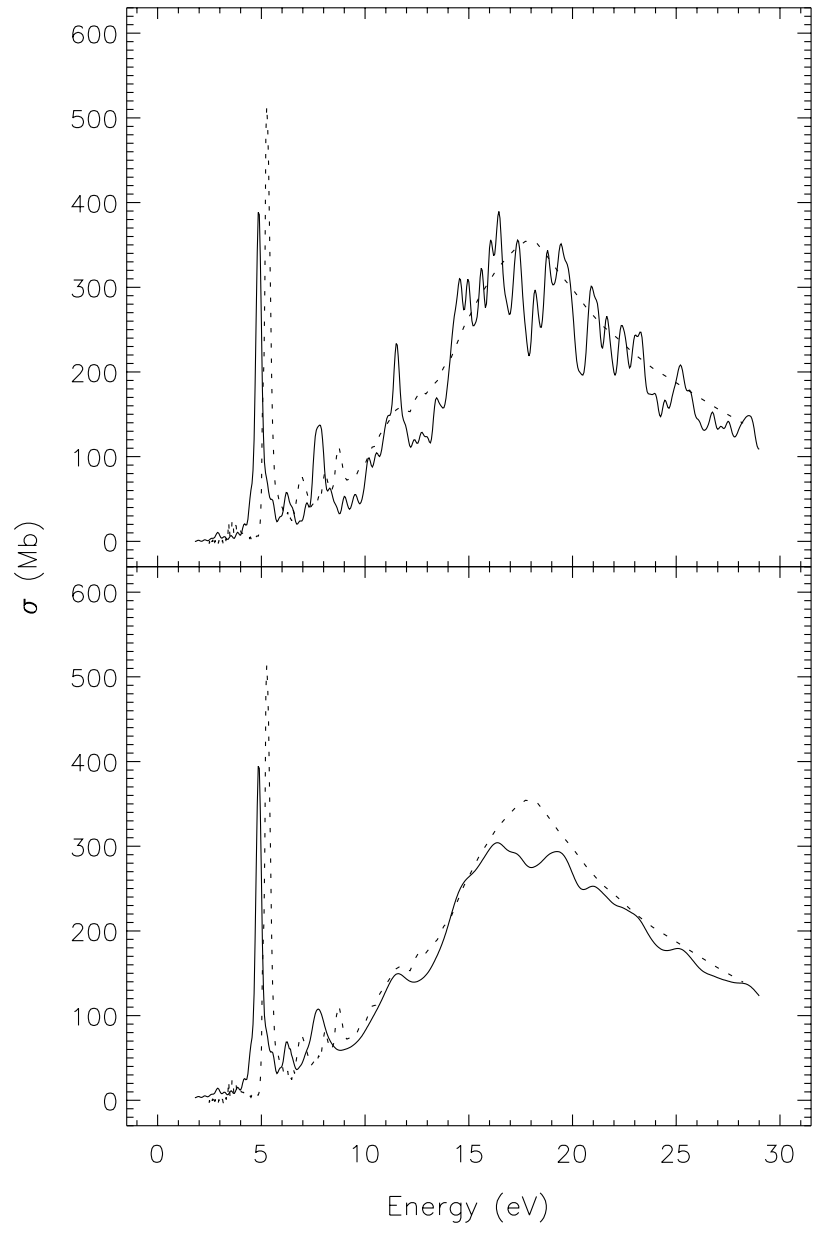

Fig. 1. Comparison between the computed (solid line) photoabsorption cross-section for neutral anthracene $\left(\mathrm{C}_{14} \mathrm{H}_{10}\right)$ and the corresponding gas-phase absorption spectrum (dotted line, from Joblin 1992; and Joblin et al. 2004). The upper panel shows the computed spectrum "as-is", i.e. just as it is produced by the octopus code; in the lower panel it was smoothed by an energy-dependent Lorentzian filter (see discussion in Sect. 2.2 for more details).

comparison with the laboratory spectrum from Joblin (1992) and Joblin et al. (2004). A detailed analysis of the experimental measurements and of their comparison with the present theoretical spectra is outside of the scope of the present paper, and will be found in a separate paper (Joblin et al. 2004).

Such a good agreement up to the VUV was unexpected, since current TD-DFT calculations are well known to be accurate and reliable for relatively low excitation energies but to increasingly underestimate the energies of more highly-excited states, the approximate empirical limit being the negative of the highest Kohn-Sham eigenvalue of the ground state (Hirata et al. 1999; Weisman et al. 2001). This is due to the wrong asymptotic behaviour of the presently available exchange-correlation functionals, which decay faster than $1 / R$ for large distances $R$ from the nuclei (see e.g., Onida et al. 2002). Despite this, Yabana \& Bertsch (1999) and Marques et al. (2003) showed theoretical spectra of benzene to compare very favorably with experimental data well up into the VUV spectral range, and we further showed this very good agreement to hold for the above mentioned sample of neutral PAHs, which validates the 
results of this theoretical approach for this class of molecules. We therefore extended these calculations to a larger sample of PAHs and their corresponding cations; concerning the latter, this study aims to shed some light on their electronic spectra, in particular in the VUV domain which is largely unknown to date.

A wide range of PAHs are thought to exist in the ISM. The total of 20 PAHs selected in this work, ranging in size from naphthalene $\left(\mathrm{C}_{10} \mathrm{H}_{8}\right)$ to dicoronylene $\left(\mathrm{C}_{48} \mathrm{H}_{20}\right)$, cover an ample range of structures inside the two large classes of pericondensed and catacondensed species. Although non-compact PAHs are expected to be less stable than compact ones with the same number of benzenoid rings, we extended the present study to both classes, including also large linear molecules such as pentacene $\left(\mathrm{C}_{22} \mathrm{H}_{14}\right)$. In addition we included some PAHs with five-membered rings, namely fluorene $\left(\mathrm{C}_{13} \mathrm{H}_{10}\right)$ and fluoranthene $\left(\mathrm{C}_{16} \mathrm{H}_{10}\right)$. We remark that the sample of small to medium-sized PAHs presented in this study has the same sizedistribution as the mixture used by Allamandola et al. (1999) to match the mid-IR spectrum of the Orion Bar. Although some studies show interstellar PAHs to be on average larger than these species (Boulanger 1999), we restricted ourselves to PAHs containing less than $\sim 50$ carbon atoms, since computational costs steeply increase for larger species (e.g. about 2100 CPU hours on an IBM SP4 for the dicoronylene cation, which was the most expensive species we studied here).

The complete set of molecules investigated, together with their orientation, are shown in Fig. 2.

\subsection{Technical details}

To obtain the electronic absorption spectrum, static Kohn-Sham wavefunctions are perturbed by an impulsive electric field, propagated for a given time interval $T$ and the absolute absorption cross-section $\sigma(\omega)$ at angular frequency $\omega$ is obtained from the time-dependent wavefunctions according to the relation,

$\sigma(\omega)=\frac{4 \pi \omega}{c} \frac{1}{3} \mathfrak{J}\{\operatorname{Tr}[\alpha(\omega)]\}=\frac{4 \pi \omega}{c} \frac{1}{3} \mathfrak{J}\left\{\sum_{i=x, y, z} \alpha_{i i}(\omega)\right\}$,

where $\mathfrak{J}$ and $\operatorname{Tr}$ represent the imaginary part and the trace of their argument respectively; $\alpha(\omega)$ is the dynamical polarisability tensor of the system and is proportional to the Fourier transform of the time-dependent dipole moment of the molecule (Castro et al. 2002; Marques et al. 2003). With this approach one always obtains the whole absorption spectrum of a given molecule, very differently to frequency-space implementations of TD-DFT, such as e.g. Gaussian and NWChem, for which computational costs scale steeply with the number of required transitions. On the other hand, the main drawback is that one does not obtain independent information for each excited state, such as the symmetry and description of the excited states, but only the direction of its transition dipole moment.

Concerning specific details of the calculations performed, we already mentioned that wavefunctions are represented by the values they take on a uniform spatial grid in a finite volume. Two important parameters of the calculations are therefore the
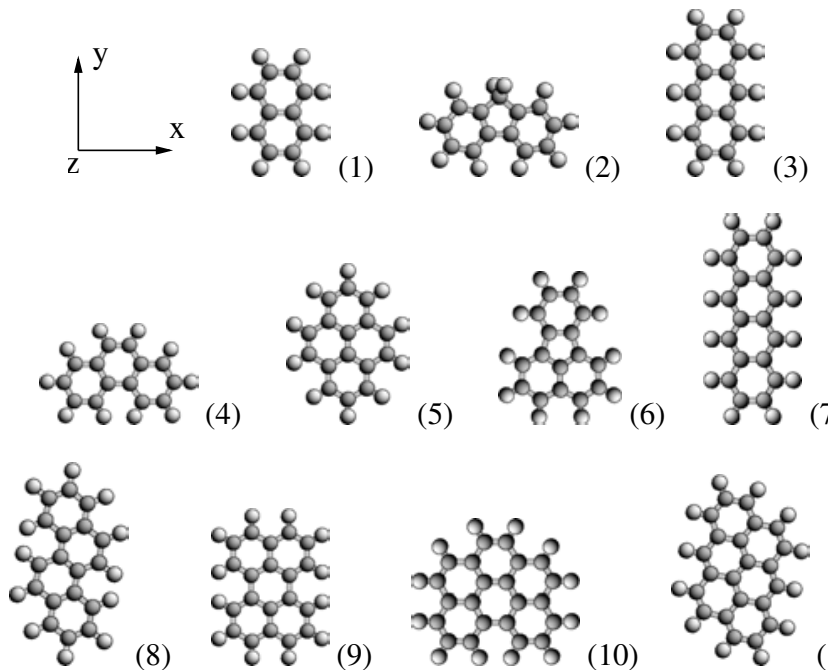

(7)

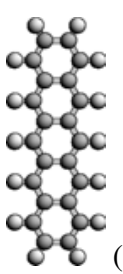

(12)

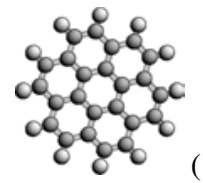

(13)

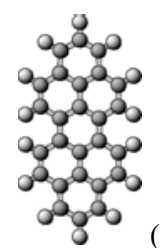

(14)
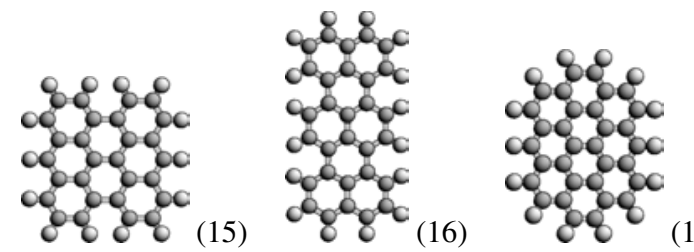

(17)
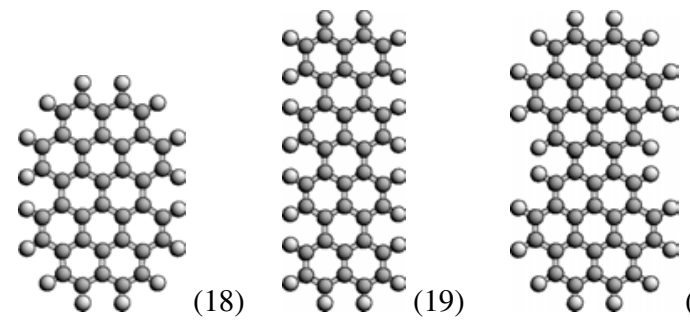

(20)

Fig. 2. Oriented structures of the PAHs investigated in this work: (1) naphthalene $\left(\mathrm{C}_{10} \mathrm{H}_{8}\right)$; (2) fluorene $\left(\mathrm{C}_{13} \mathrm{H}_{10}\right)$; (3) anthracene $\left(\mathrm{C}_{14} \mathrm{H}_{10}\right)$; (4) phenanthrene $\left(\mathrm{C}_{14} \mathrm{H}_{10}\right)$; (5) pyrene $\left(\mathrm{C}_{16} \mathrm{H}_{10}\right)$; (6) fluoranthene $\left(\mathrm{C}_{16} \mathrm{H}_{10}\right)$; (7) tetracene $\left(\mathrm{C}_{18} \mathrm{H}_{12}\right)$; (8) chrysene $\left(\mathrm{C}_{18} \mathrm{H}_{12}\right)$; (9) perylene $\left(\mathrm{C}_{20} \mathrm{H}_{12}\right)$; (10) benzo[g,h,i]perylene $\left(\mathrm{C}_{22} \mathrm{H}_{12}\right)$; (11) anthanthrene $\left(\mathrm{C}_{22} \mathrm{H}_{12}\right)$; (12) pentacene $\left(\mathrm{C}_{22} \mathrm{H}_{14}\right)$; (13) coronene $\left(\mathrm{C}_{24} \mathrm{H}_{12}\right)$; (14) dibenzo[cd,lm]perylene $\left(\mathrm{C}_{26} \mathrm{H}_{14}\right)$; (15) bisanthene $\left(\mathrm{C}_{28} \mathrm{H}_{14}\right)$; (16) terrylene $\left(\mathrm{C}_{30} \mathrm{H}_{16}\right)$; (17) ovalene $\left(\mathrm{C}_{32} \mathrm{H}_{14}\right)$; (18) circumbiphenyl $\left(\mathrm{C}_{38} \mathrm{H}_{16}\right)$; (19) quaterrylene $\left(\mathrm{C}_{40} \mathrm{H}_{20}\right)$; (20) dicoronylene $\left(\mathrm{C}_{48} \mathrm{H}_{20}\right)$.

volume of the box in which the molecule is represented, and the spatial resolution $\Delta s$ of the grid. Following the calibrations performed for carbon-hydrogen systems by Yabana \& Bertsch (1999), we used a grid spacing of $0.3 \AA$ (or sligthy smaller for those systems whose absorption spectrum showed minor convergence problems), and determined the box size by requiring each atom to be at least a few $\AA$ away from its edges (typically 3-4 $\AA$ are sufficient). We chose a time integration length of $20 \hbar / \mathrm{eV}$, which corresponds to an energy resolution of 
$\hbar / T=0.05 \mathrm{eV}$, much more precise than the accuracy of current TD-DFT results for such molecules (Hirata et al. 1999). The time evolution was performed using a time step of $0.001 \hbar / \mathrm{eV}$, which ensured energy conservation with extremely good numerical accuracy, namely better than one part in a million.

Since octopus is not optimized to obtain the groundstate geometries, we decided to obtain the equilibrium configurations of the studied species through the Gaussianbased DFT module of the NWChem computer code (Straatsma et al. 2003). More specifically, following Langhoff (1996), Bauschlicher \& Langhoff (1997), and Hudgins et al. (2001) we decided to use for this purpose the hybrid exchange-correlation functional B3LYP (Becke 1993; Stephens et al. 1994) and the 4-31G Gaussian basis set (Frish et al. 1984) to expand the molecular orbitals. The optimized ground state structures were always planar (with the sole exception of fluorene) and the corresponding molecular symmetry was assumed during calculations (e.g. $\mathrm{D}_{2 h}$ for ovalene, $\mathrm{C}_{2 v}$ for phenanthrene, $\mathrm{C}_{2 h}$ for chrysene, and so forth), with the exception of the more symmetric coronene, belonging to $\mathrm{D}_{6 h}$ point group, for whose cation the lifting of symmetry restrictions leads to optimized geometries with the lower symmetry $\mathrm{D}_{2 h}$, as expected due to Jahn-Teller distortion (Weisman et al. 2003). To confirm the obtained geometry to be indeed a minimum on the potential energy hypersurface, we also performed a full vibrational analysis which always yielded real values for every computed eigen-frequency. For all the species presented here, the complete list of frequencies, the corresponding symmetries, and the absolute intensities of the corresponding 0-1 transitions of the IR-active modes are available at http://www . ars-network.org.

Although Gaussian basis sets larger than 4-31G are available in NWChem, their use results in changes never larger than a few percent in structural parameters, hence the resulting electronic absorption spectrum, as computed by octopus, is largely unaffected. Moreover, the choice of the relatively small basis set 4-31G arises from well established calibration studies (Langhoff 1996; Bauschlicher \& Langhoff 1997), showing the B3LYP/4-31G level of theory, together with the use of a single empirical scaling factor, to be particularly accurate for the computation of the vibrational frequencies of PAHs (Langhoff 1996; Bauschlicher \& Langhoff 1997; Hudgins et al. 2001; Bauschlicher Jr. 2002), bringing them into near coincidence with the available experimental data. Indeed, we already used the same approach in two recent papers (Malloci et al. 2003; Mulas et al. 2003) where our calculated vibrational frequencies for the ovalene $\left(\mathrm{C}_{32} \mathrm{H}_{14}\right)$ neutral and cation where shown to be in good agreement with the results previously published by Langhoff (1996).

We remark that the approach we used yields vertical, purely electronic spectra, at a frozen ionic configuration. Only boundbound electronic transitions are included in this approach, but bound-free transitions (i.e. direct ionisations) are expected to become important at energy significantly higher than those considered here. In experimental spectra, there is usually a sequence of vibronic transitions instead of a single band, and each transition is substantially broadened by the fast decay of excited states due to internal conversion. These effects are not considered in the calculations. The broadening becomes

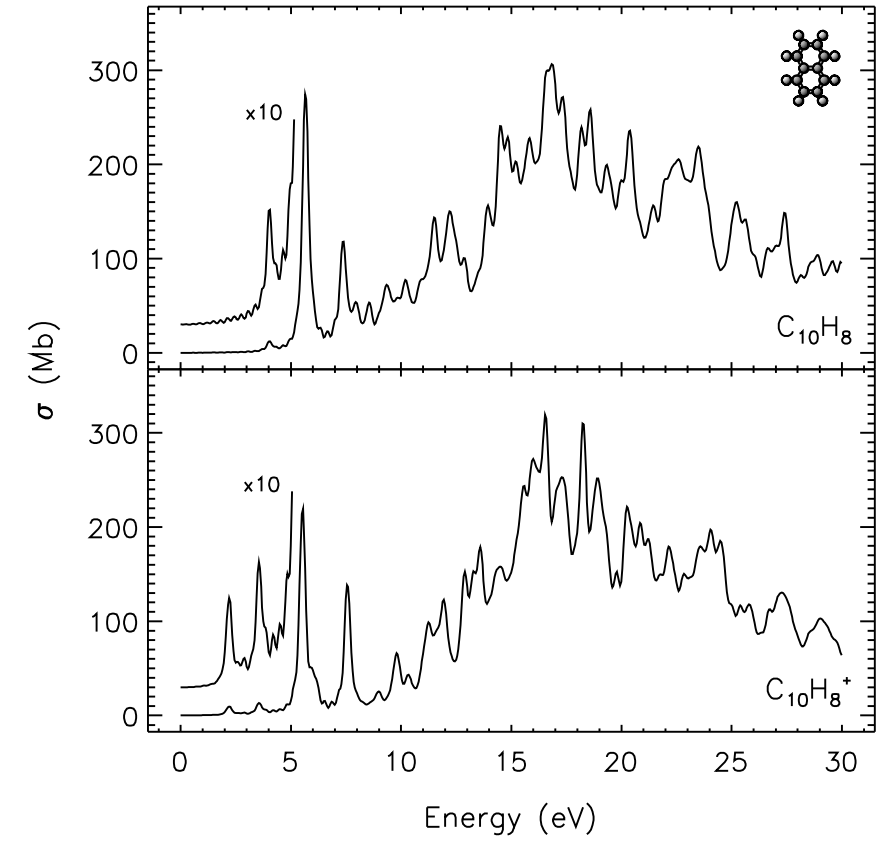

Fig. 3. Computed photo-absorption cross-section of naphthalene $\left(\mathrm{C}_{10} \mathrm{H}_{8}\right)$ neutral and cation. Units are megabarns $\left(1 \mathrm{Mb}=10^{-18} \mathrm{~cm}^{2}\right)$.

increasingly important with increasing energy of the excited electronic state involved in the transition, and causes the relatively sharp bands visible in the calculated far-UV spectrum to blend in a broad, featureless bump in laboratory data. To obtain synthetic spectra as close as possible to the true experimental ones, one may apply an energy-dependent broadening to the output of octopus, tuning the parameters of the energydependent broadening on the molecules for which laboratory data are available. The result of such a procedure is shown in Fig. 1. For these reasons, the fine structure visible in the theoretical spectra above $\sim 10 \mathrm{eV}$ ought to be disregarded and only the overall behaviour should be considered as real.

\section{Results and discussion}

Figures 3 through 22 present the computed spectra for each of the studied PAHs and its corresponding cation. Photoabsorption cross-sections are expressed in megabarns $(1 \mathrm{Mb}=$ $10^{-18} \mathrm{~cm}^{2}$ ) and the spectra are displayed up to $\sim 30 \mathrm{eV}$. As explained in Sect. 2, our present results are validated by the good agreement with available experimental data found both in the low-energy range, where TD-DFT is known to work quite well, and in the VUV domain, as shown in the above Fig. 1 and discussed in more detail in Joblin et al. (2004). Table 1 shows that the octopus approach used here is as accurate as previously published theoretical results on low-energy transitions, when compared with available experimental data.

The broad band at $\sim 17-18 \mathrm{eV}$ deserves some comment in its own right. The good overall agreement between our TD-DFT spectra and experimental data implies that this theory can account, for this specific class of molecules, for the excited electronic states which can be reached via electric dipole permitted transitions from the ground electronic state. This band 
Table 1. Singlet $\pi^{*} \leftarrow \pi$ excitations of a subset of PAH cations in our sample. Our calculated excitation energies (in eV) and oscillator strengths (in parentheses) are compared to available experimental data as well as with published TD-DFT results. The direction of the transition dipole for each band is given according to the molecular geometries sketched in Fig. 2. Ground state geometries are used in all calculations, which therefore refer to vertical electronic transitions, expected to occur at slightly larger energies than the experimentally measured non-vertical transitions.

\begin{tabular}{|c|c|c|c|}
\hline $\begin{array}{c}\text { Dipole } \\
\text { orientation }\end{array}$ & $\begin{array}{l}\text { This } \\
\text { work }\end{array}$ & $\begin{array}{l}\text { Published } \\
\text { theoretical }\end{array}$ & $\begin{array}{c}\text { Published } \\
\text { experimental }\end{array}$ \\
\hline \multicolumn{4}{|c|}{ Naphthalene cation $\left(\mathrm{C}_{10} \mathrm{H}_{8}^{+}\right)$} \\
\hline$y$ & $2.20(0.047)$ & $2.35(0.057)^{a}$ & $\begin{array}{c}1.85(0.052)^{b} \\
1.85(/)^{c}, 1.85(/)^{d}\end{array}$ \\
\hline$x$ & $2.82(0.006)$ & $2.85(0.007)^{a}$ & $2.72(0.010)^{b}$ \\
\hline \multicolumn{4}{|c|}{ Anthracene cation $\left(\mathrm{C}_{14} \mathrm{H}_{10}^{+}\right)$} \\
\hline$y$ & $1.85(0.082)$ & $2.02(0.116)^{a}$ & $\begin{array}{c}1.72(0.076)^{e} \\
1.75(/)^{f}\end{array}$ \\
\hline$x$ & $2.88(0.039)$ & $3.06(0.067)^{a}$ & $2.90(0.026)^{e}$ \\
\hline \multicolumn{4}{|c|}{ Pyrene cation $\left(\mathrm{C}_{16} \mathrm{H}_{10}^{+}\right)$} \\
\hline$x$ & $1.59(0.015)$ & $1.64(0.017)^{g}$ & $1.58(0.005)^{h}$ \\
\hline$y$ & $2.94(0.204)$ & $2.98(0.219)^{g}$ & $\begin{array}{l}2.79(0.100)^{h} \\
2.84(/)^{d}\end{array}$ \\
\hline \multicolumn{4}{|c|}{ Perylene cation $\left(\mathrm{C}_{20} \mathrm{H}_{12}^{+}\right)$} \\
\hline$x$ & $1.89(0.034)$ & $1.92(0.029)^{i}$ & $1.93(\mathrm{w})^{l}$ \\
\hline$y$ & $2.38(0.326)$ & $2.44(0.348)^{i}$ & $2.36(/)^{i}, 2.32(\mathrm{~s})^{l}$ \\
\hline \multicolumn{4}{|c|}{ Pentacene cation $\left(\mathrm{C}_{22} \mathrm{H}_{14}^{+}\right)$} \\
\hline$x$ & $1.17(0.007)$ & $1.26(0.012)^{m}$ & $1.27(/)^{m}$ \\
\hline$y$ & $1.41(0.163)$ & $1.51(0.229)^{m}$ & $1.31(/)^{m}$ \\
\hline \multicolumn{4}{|c|}{ Terrylene cation $\left(\mathrm{C}_{30} \mathrm{H}_{16}^{+}\right)$} \\
\hline$x$ & $1.82(0.019)$ & $1.83(0.014)^{i}$ & / \\
\hline$y$ & $1.88(0.667)$ & $1.93(0.706)^{i}$ & $1.80(/)^{i}$ \\
\hline \multicolumn{4}{|c|}{ Ovalene cation $\left(\mathrm{C}_{32} \mathrm{H}_{14}^{+}\right)$} \\
\hline$y$ & $1.23(0.048)$ & $1^{14}$ & $1.27(/)^{n}$ \\
\hline$x$ & $2.14(0.138)$ & $2.15(0.141)^{i}$ & $2.21(/)^{n}$ \\
\hline \multicolumn{4}{|c|}{ Quaterrylene cation $\left(\mathrm{C}_{40} \mathrm{H}_{20}^{+}\right)$} \\
\hline$y$ & $1.58(1.044)$ & $1.62(1.028)^{i}$ & $1.48(/)^{i}$ \\
\hline$x$ & $1.79(0.021)$ & $1.81(0.018)^{i}$ & 1 \\
\hline \multicolumn{4}{|c|}{ Dicoronylene cation $\left(\mathrm{C}_{48} \mathrm{H}_{20}^{+}\right)$} \\
\hline$y$ & $1.17(0.155)$ & / & $1.21(/)^{o}$ \\
\hline$y$ & $1.87(0.683)$ & 1 & $1.82(/)^{o}$ \\
\hline
\end{tabular}

${ }^{a}$ TD-DFT calculation by Hirata et al. (1999).

${ }^{c}$ Gas-phase absorption spectrum from Pino et al. (1999).

${ }^{c}$ Gas-phase absorption spectrum from Romanini et al. (1999).

${ }^{d}$ Gas-phase absorption spectrum from Biennier et al. (2004).

${ }^{e}$ Ar-matrix absorption spectrum from Szczepanski et al. (1993b).

${ }^{f}$ Gas-phase absorption spectrum from Sukhorukov et al. (2004).

${ }^{g}$ TD-DFT calculation by Weisman et al. (2003).

${ }^{h}$ Ar-matrix absorption spectrum from Vala et al. (1994).

${ }^{i}$ TD-DFT calculations and Ne-matrix absorption spectrum from Halasinski et al. (2003).

${ }^{l}$ Ar-matrix absorption spectrum from Szczepanski et al. (1993a).

$m$ TD-DFT calculations and Ne-matrix absorption spectrum from Halasinski et al. (2000).

${ }^{n}$ Ne-matrix absorption spectrum from Ehrenfreund et al. (1992).

${ }^{o}$ Ne-matrix absorption spectrum from Ruiterkamp et al. (2002).

has been attributed mainly to $\sigma^{*} \leftarrow \sigma$ transitions by Verstraete \& Léger (1992), with possible reinforcement of its intensity by

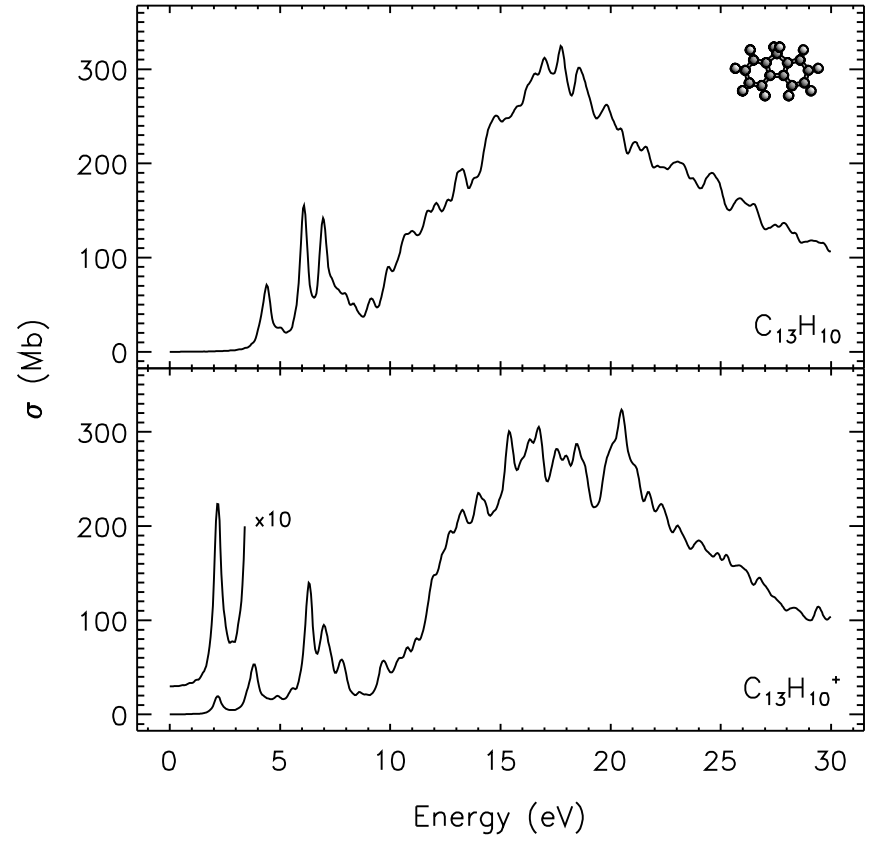

Fig. 4. Same as Fig. 3 for fluorene $\left(\mathrm{C}_{13} \mathrm{H}_{10}\right)$ neutral and cation.

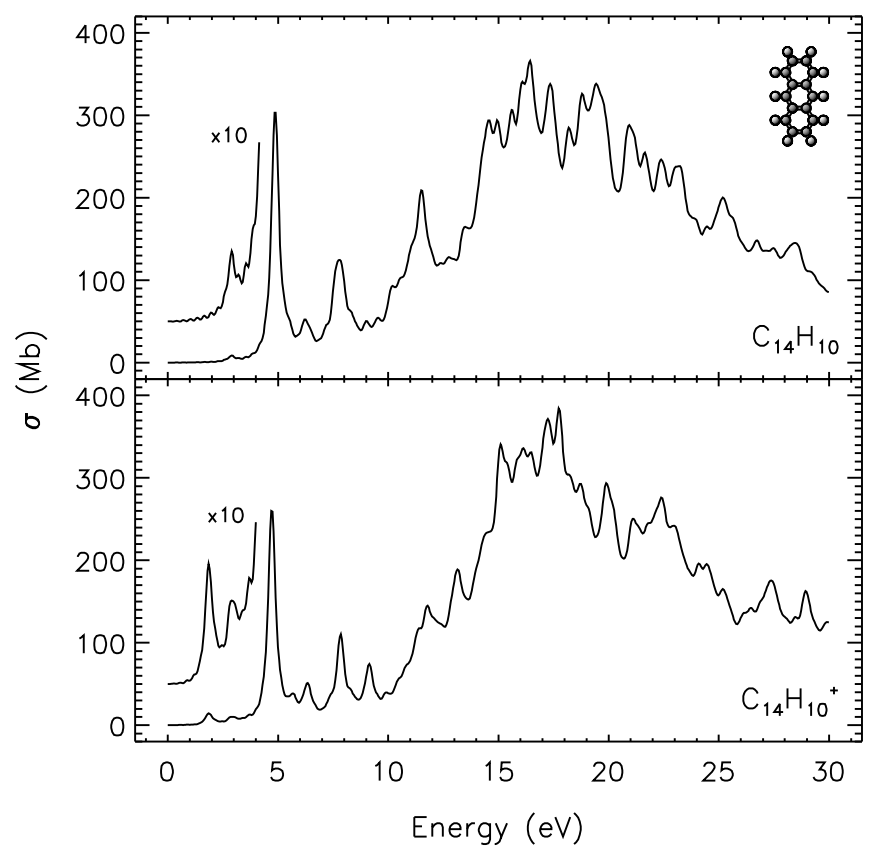

Fig. 5. Same as Fig. 3 for anthracene $\left(\mathrm{C}_{14} \mathrm{H}_{10}\right)$ neutral and cation.

collective effects (Jochims et al. 1997). Leach (1995) showed that $\sigma^{*} \leftarrow \sigma$ transitions ought to be largely unaffected by ionisation, hence the striking similarity we obtain between the VUV spectrum of each cation with its parent neutral is consistent with the attribution of the broad band to these transitions, with no strong need for collective effects.

Moreover, it is worth noting that the TD-DFT implementation we used, with a finite simulation box, utterly neglects transitions to unbound electronic states, i.e. transitions leading to direct ionisation. The good agreement in Fig. 1 implies that transitions to superexcited states account for almost all of the absorption cross section. On the other hand, the 


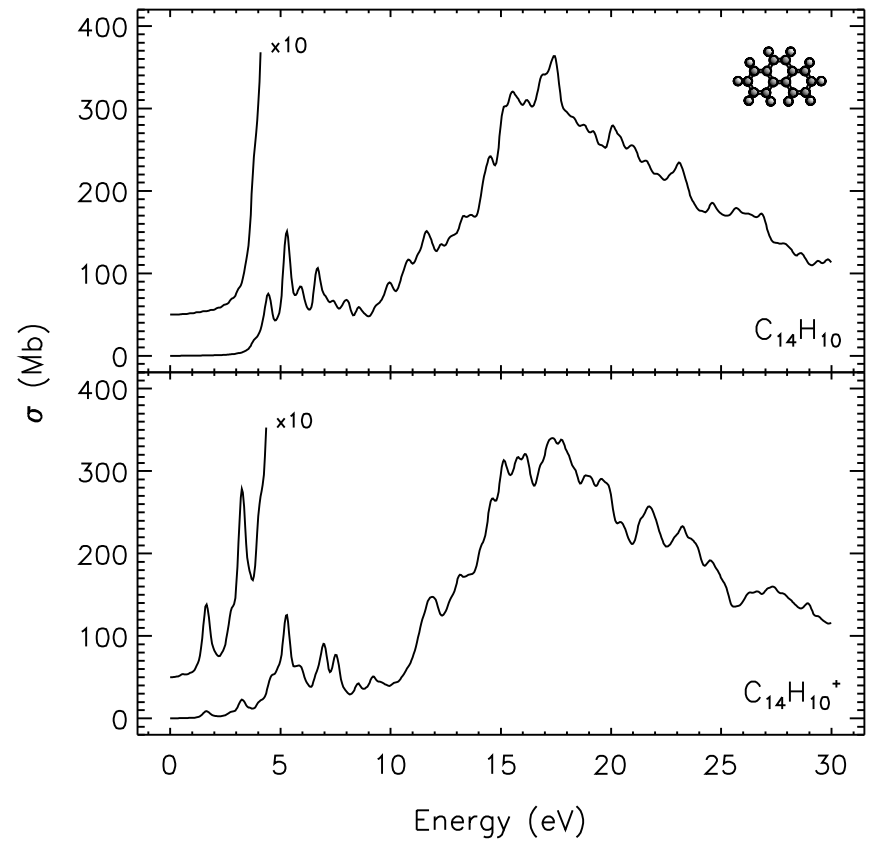

Fig. 6. Same as Fig. 3 for phenanthrene $\left(\mathrm{C}_{14} \mathrm{H}_{10}\right)$ neutral and cation.

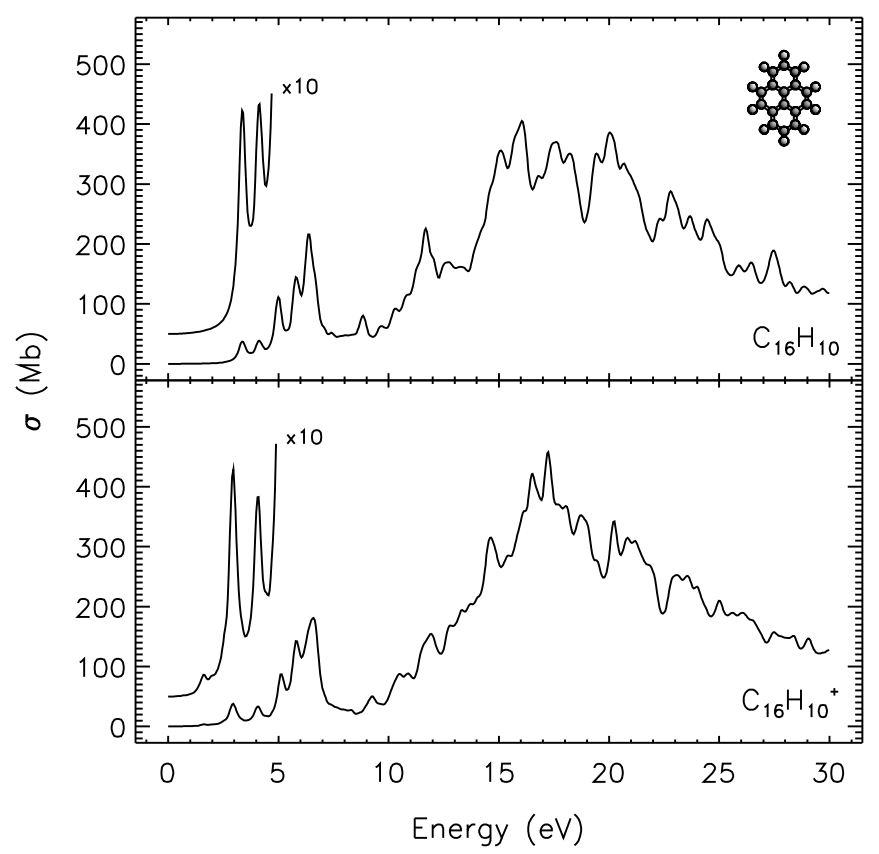

Fig. 7. Same as Fig. 3 for pyrene $\left(\mathrm{C}_{16} \mathrm{H}_{10}\right)$ neutral and cation.

ionisation yield was measured to be $100 \%$ at $\sim 14 \mathrm{eV}$ for neutral pyrene $\left(\mathrm{C}_{16} \mathrm{H}_{10}\right)$ and coronene $\left(\mathrm{C}_{24} \mathrm{H}_{12}\right)$ (Verstraete et al. 1990). This confirms that superexcited states are coupled to the ionisation continuum. Jochims et al. (1997) concluded that this is effective at energies higher than $\sim 15 \mathrm{eV}$ for PAHs with ionisation potential in the range $\sim 6.5-9 \mathrm{eV}$.

More generally, our results confirm some well known trends in the absorption properties of small to medium-sized PAHs, which have been recently reviewed by Salama (1999):

- small neutral PAHs (with less than about 20-25 carbon atoms) do not absorb in the visible-near infrared range

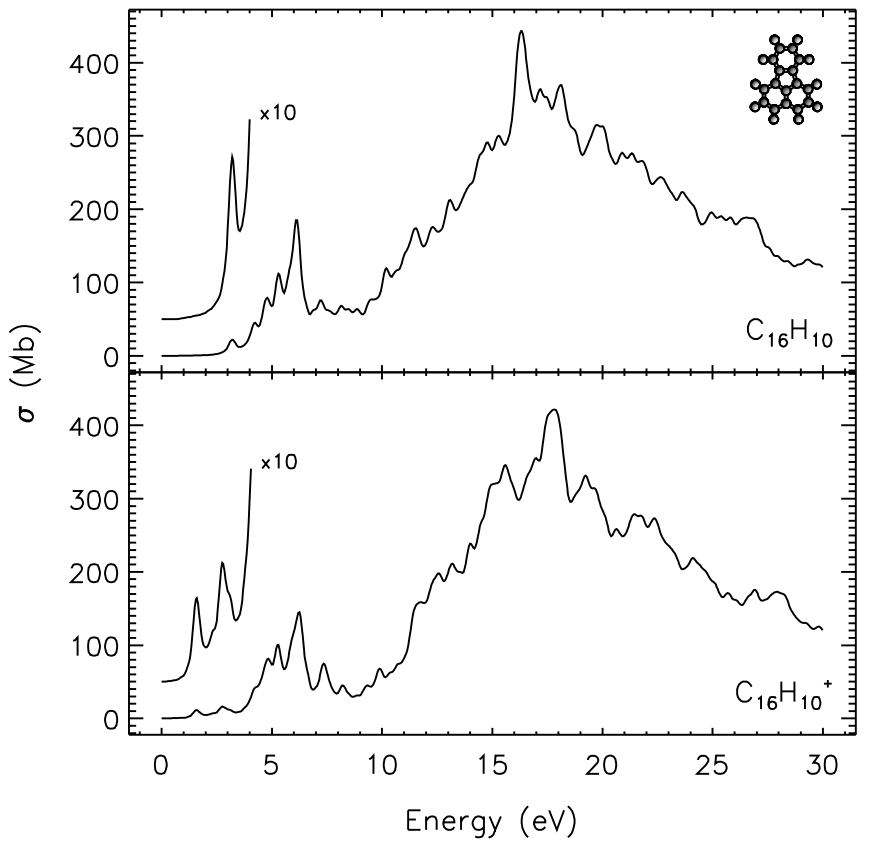

Fig. 8. Same as Fig. 3 for fluoranthene $\left(\mathrm{C}_{16} \mathrm{H}_{10}\right)$ neutral and cation.

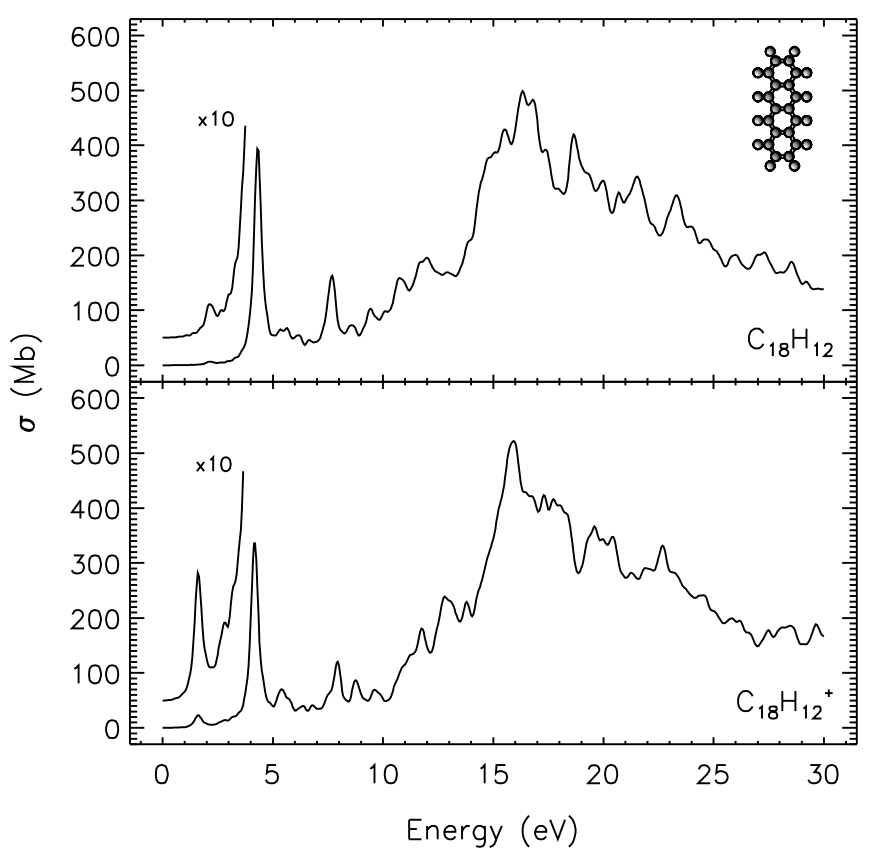

Fig. 9. Same as Fig. 3 for tetracene $\left(\mathrm{C}_{18} \mathrm{H}_{12}\right)$ neutral and cation.

but display strong UV absorption bands, while their respective cations do absorb in the visible;

- the position of the lowest-lying absorption band for neutral PAHs shifts towards lower energies as the size of the molecule increases;

- a single strong band dominates the absorption spectrum of most PAH cations in the spectral region from the near-UV to the near-IR.

The most interesting result, however, is the UV-VUV spectrum of PAH cations since, to the best of our knowledge, a detailed discussion of the spectral properties of PAH ions in this 


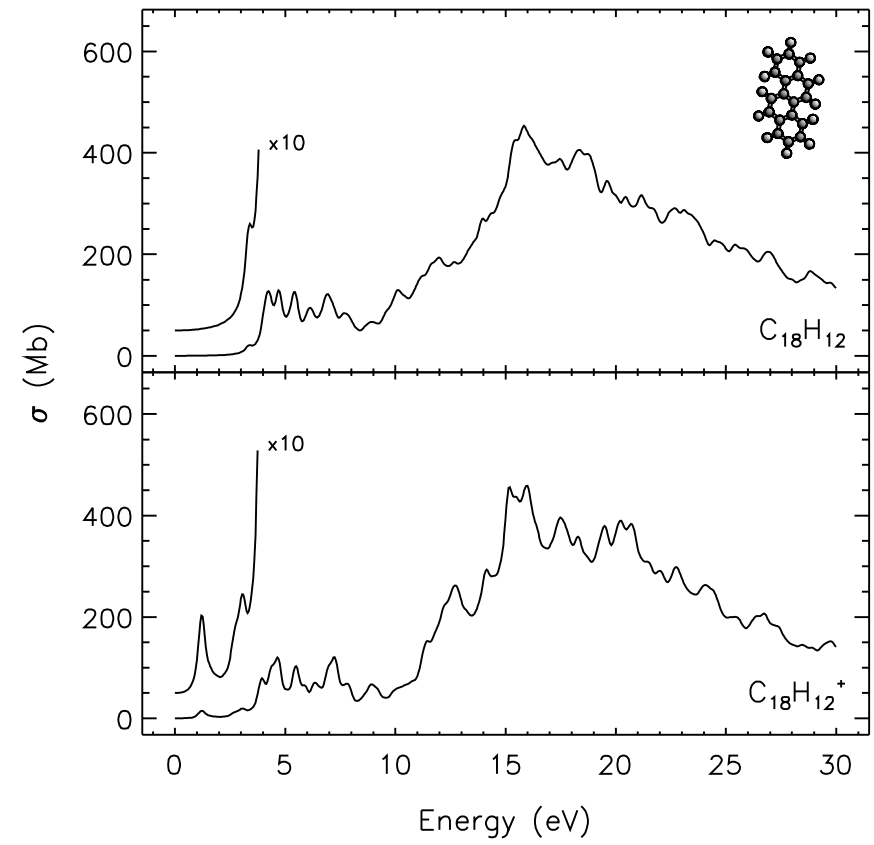

Fig. 10. Same as Fig. 3 for chrysene $\left(\mathrm{C}_{18} \mathrm{H}_{12}\right)$ neutral and cation.

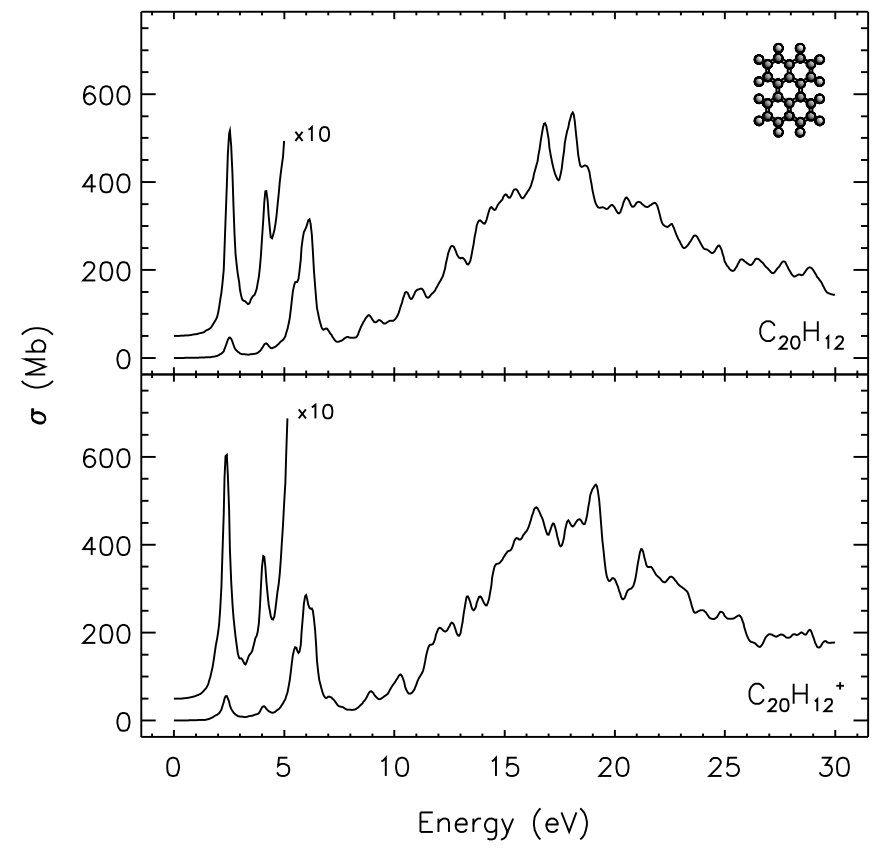

Fig. 11. Same as Fig. 3 for perylene $\left(\mathrm{C}_{20} \mathrm{H}_{12}\right)$ neutral and cation.

domain has been missing until now. In this range the spectra of the cations and parent neutrals appear to be very close and it is very difficult to disentangle the contributions of the two charge states to the bands. The first detection of an ultraviolet transition in an ionized $\mathrm{PAH}$ has been reported by Chillier et al. (1999) for perylene cation $\left(\mathrm{C}_{20} \mathrm{H}_{12}{ }^{+}\right)$. Before the above experimental evidence, this point had been debated for some years, and in astronomical models the absorption properties of cations were usually obtained from an educated guess assuming the spectrum of the ion:

1. to have no strong $\pi^{*} \leftarrow \pi$ transitions in the near UV (Lee \& Wdowiak 1993; Robinson et al. 1997);

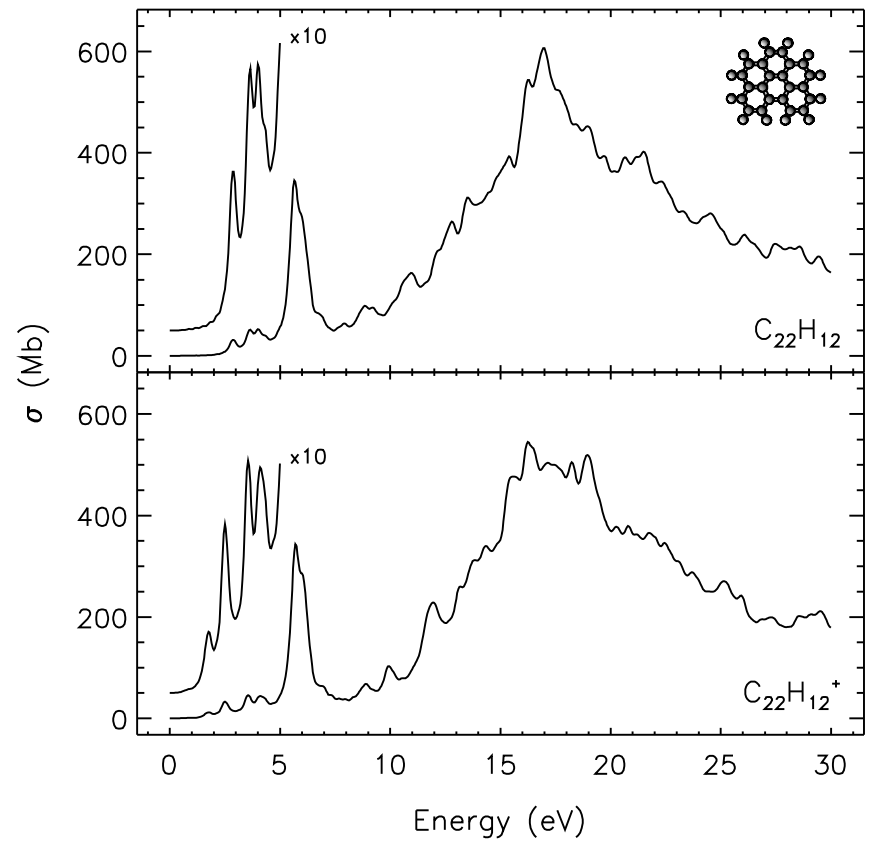

Fig. 12. Same as Fig. 3 for benzo[g,h,i]perylene $\left(\mathrm{C}_{22} \mathrm{H}_{12}\right)$ neutral and cation.

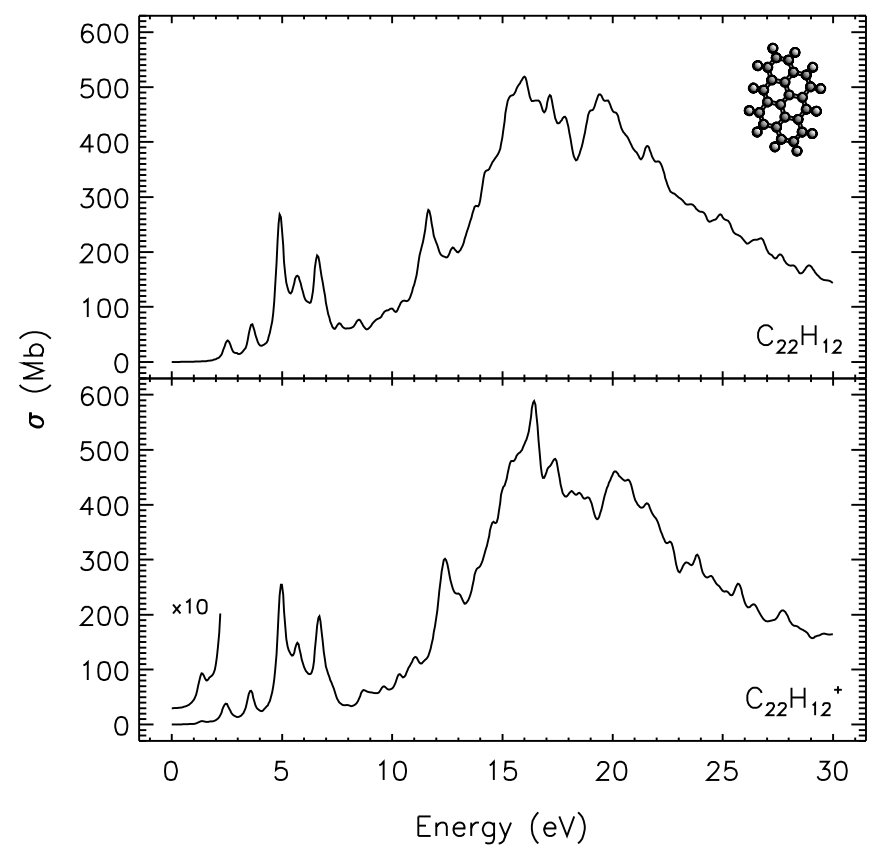

Fig. 13. Same as Fig. 3 for anthanthrene $\left(\mathrm{C}_{22} \mathrm{H}_{12}\right)$ neutral and cation.

2. to resemble the spectrum of its parent neutral for the higher excited states, since $\sigma^{*} \leftarrow \sigma$ transitions are expected to be largely unaffected by ionisation (Leach 1995).

More specifically, such cations were assumed to possess no strong $\pi^{*} \leftarrow \pi$ transitions up to about $7.75 \mathrm{eV}$. The above energy limit had been inferred from the absorption spectra taken by Robinson et al. (1997).

Both the experimental work of Chillier et al. (1999) and theoretical calculations (Weisman et al. 2003) strongly suggested the above simplistic assumption 1 to be invalid: large PAHs must approach the behaviour of small dust particles, 


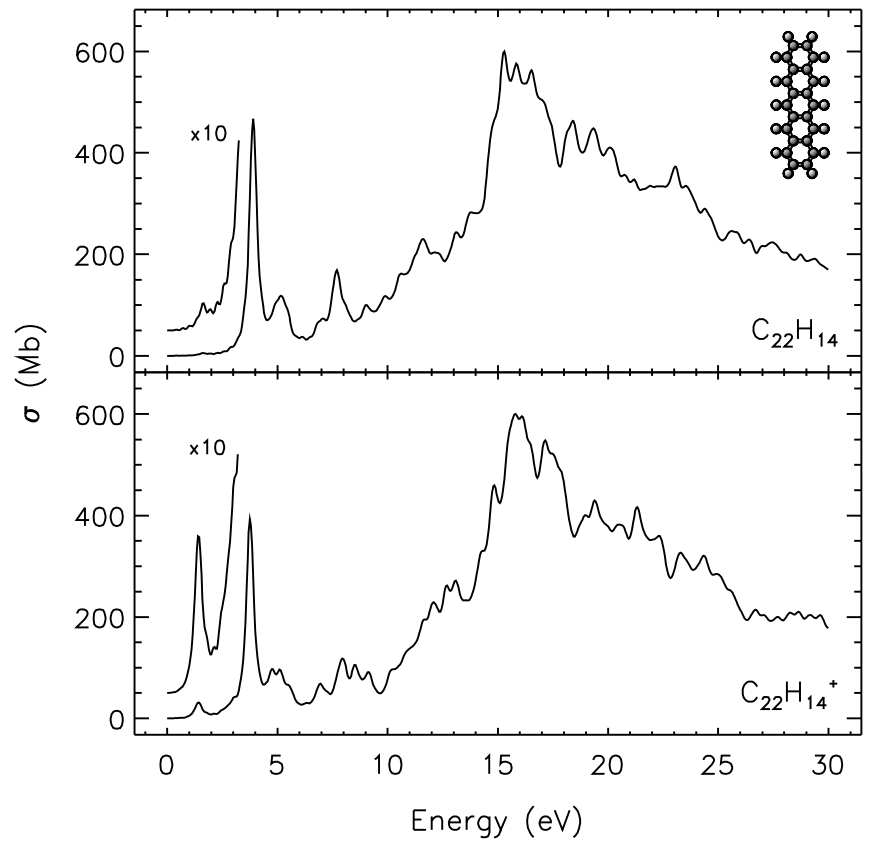

Fig. 14. Same as Fig. 3 for pentacene $\left(\mathrm{C}_{22} \mathrm{H}_{14}\right)$ neutral and cation.

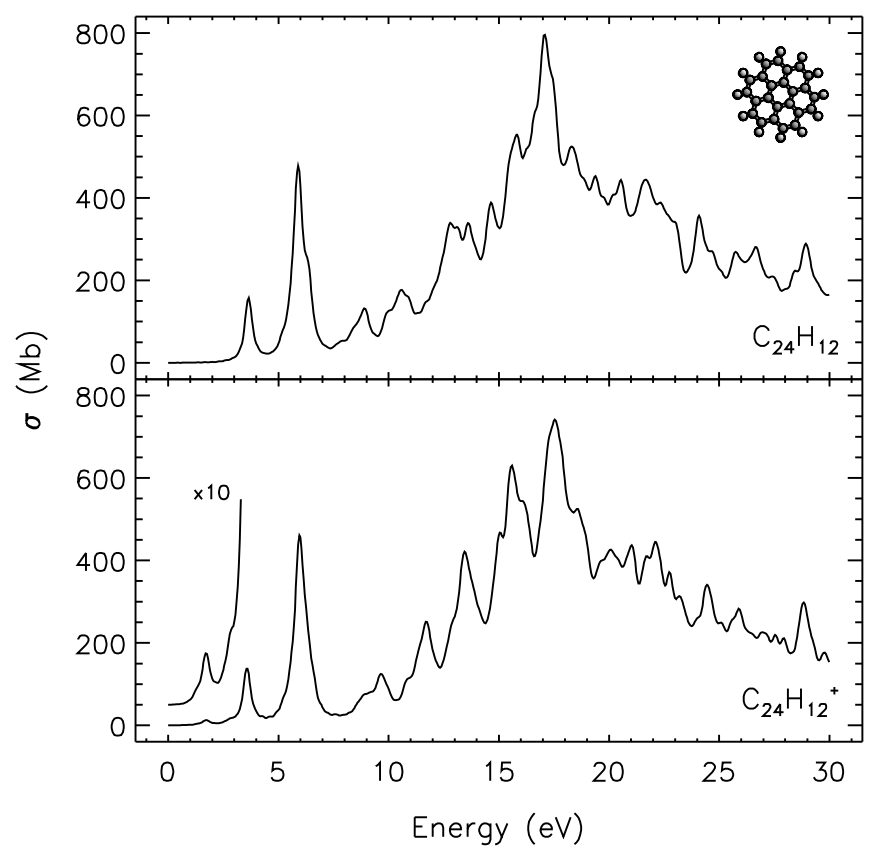

Fig. 15. Same as Fig. 3 for coronene $\left(\mathrm{C}_{24} \mathrm{H}_{12}\right)$ neutral and cation.

whose spectrum is obviously almost unaffected by the loss of a single electron. Our results do confirm on a systematic basis assumption 2 to be valid and assumption 1 to be invalid across the sample studied here. The spectrum we calculated for each PAH cation is remarkably similar to the one of its parent neutral molecule, being dominated by almost as strong $\pi^{*} \leftarrow \pi$ transitions in the UV range.

\section{Astrophysical implications}

With respect to the spectral range covering the near-IR, visible and near-UV, it is obvious that with more than 300 DIBs

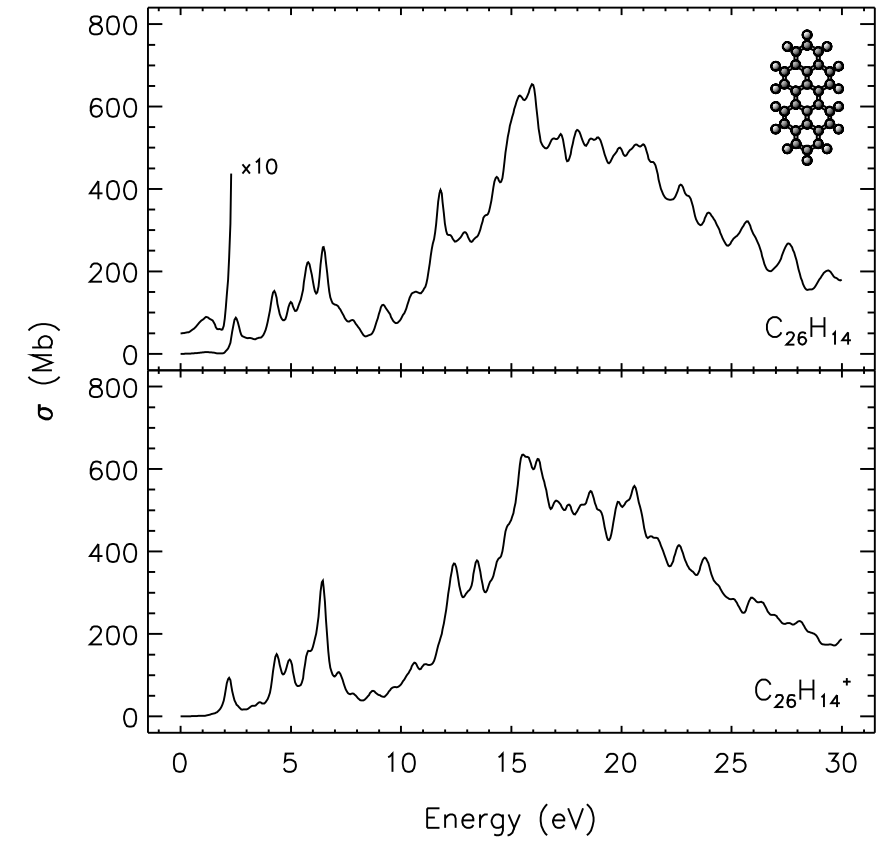

Fig. 16. Same as Fig. 3 for dibenzo[cd,lm]perylene $\left(\mathrm{C}_{26} \mathrm{H}_{14}\right)$ neutral and cation.

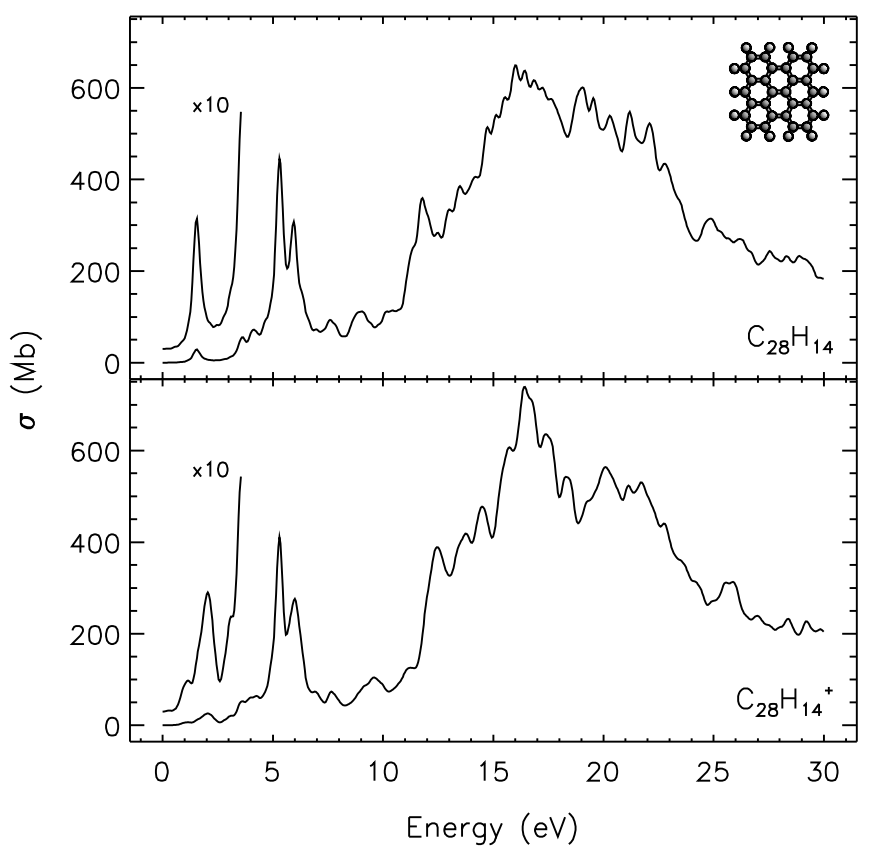

Fig. 17. Same as Fig. 3 for bisanthene $\left(\mathrm{C}_{28} \mathrm{H}_{14}\right)$ neutral and cation.

in this window the accuracy of about $0.3 \mathrm{eV}$ achieved by current TD-DFT methods on the position of the bands cannot be used alone for a firm spectral identification. Still, theoretical spectra in this range are an useful tool both to guide future experiments (Weisman et al. 2003) and to interpret laboratory spectra as they become available (Halasinski et al. 2003). Since there is already an ample literature about low-energy transitions of neutral and cationic PAHs (see e.g., Salama et al. 1996, 1999; Bréchignac \& Pino 1999), a thorough description of the low-lying spectral transitions is beyond the scope of this paper. 


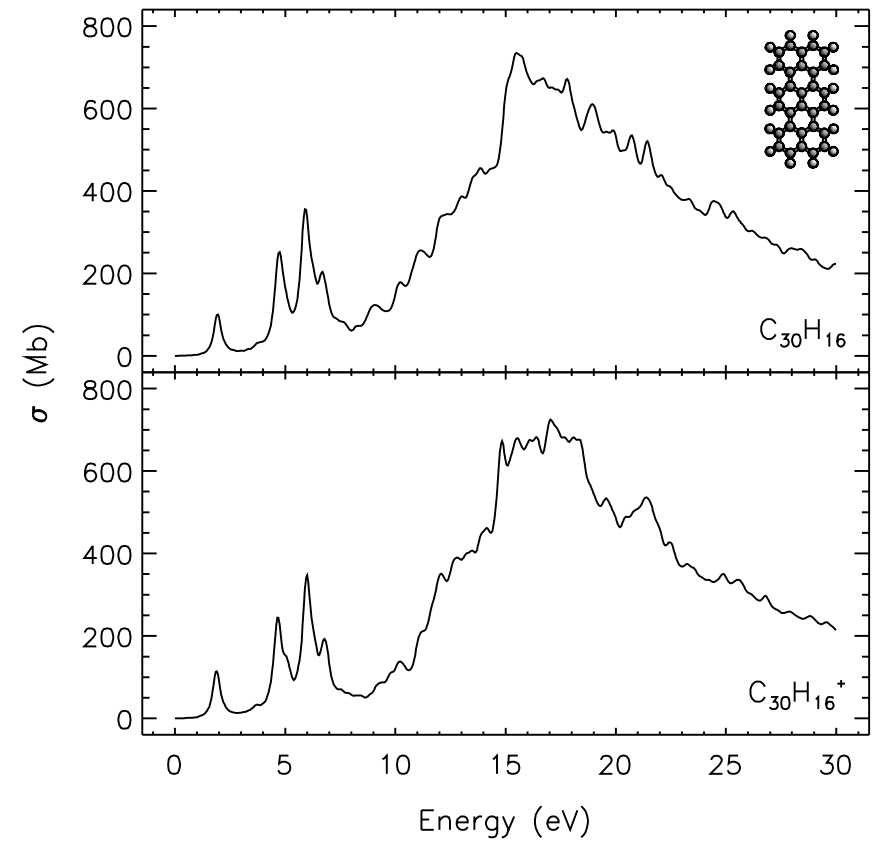

Fig. 18. Same as Fig. 3 for terrylene $\left(\mathrm{C}_{30} \mathrm{H}_{16}\right)$ neutral and cation.

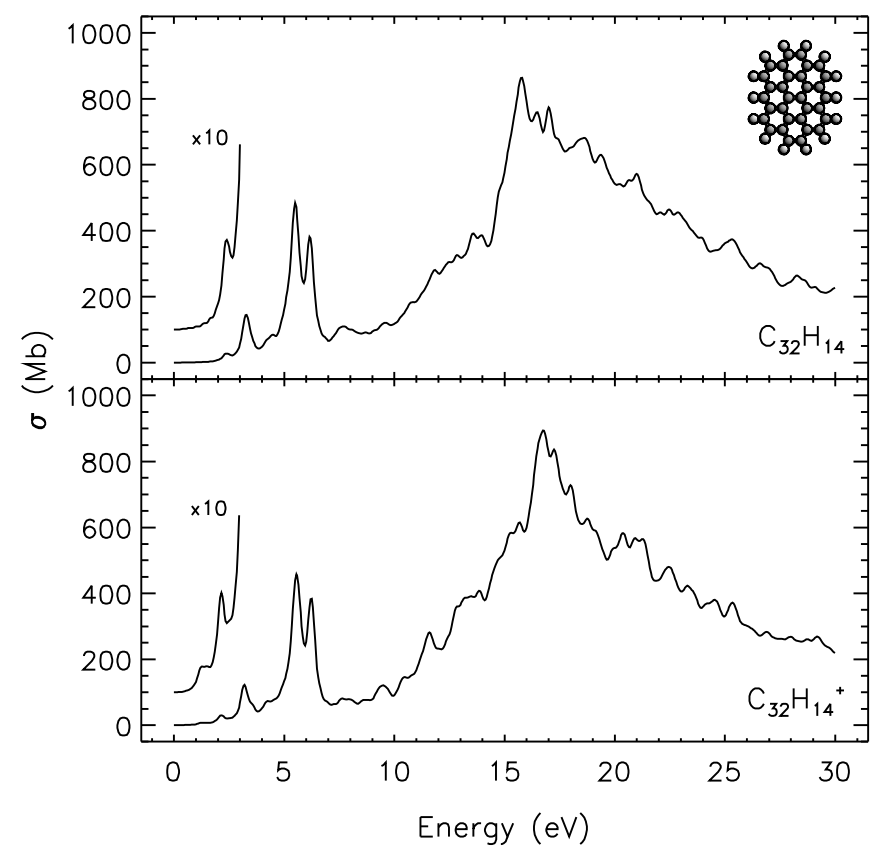

Fig. 19. Same as Fig. 3 for ovalene $\left(\mathrm{C}_{32} \mathrm{H}_{14}\right)$ neutral and cation.

We henceforth concentrate our discussion on the UV range since the most important new results presented here are the electronic absorption properties of small to medium-sized PAHs in this spectral domain. In the past, several authors noted that if neutral PAHs were to be responsible for the AIBs, their UV spectroscopic signatures ought to be detectable on the interstellar extinction curve (Donn et al. 1989; Leach 1995). This was not taken as a serious drawback of the PAH hypothesis, since they were expected to be mostly ionised in the diffuse ISM and their cations were assumed to have no sharp features in the near-UV (Lee \& Wdowiak 1993; Robinson et al. 1997). The work by Chillier et al. (1999) and by Weisman et al. (2003)

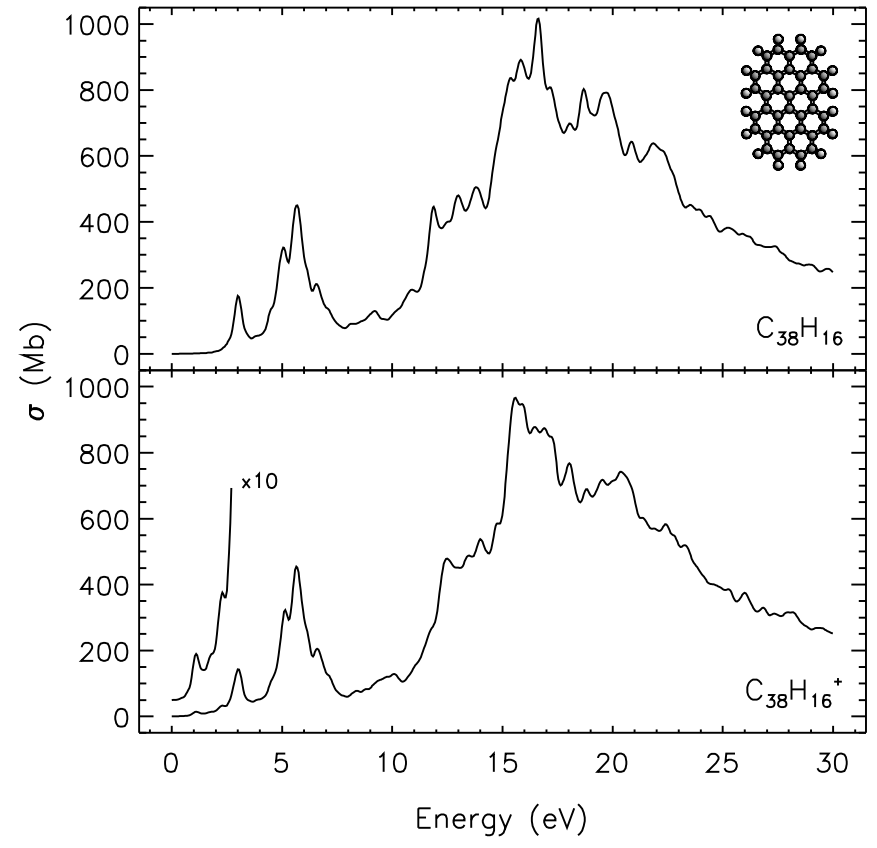

Fig. 20. Same as Fig. 3 for circumbiphenyl $\left(\mathrm{C}_{38} \mathrm{H}_{16}\right)$ neutral and cation.

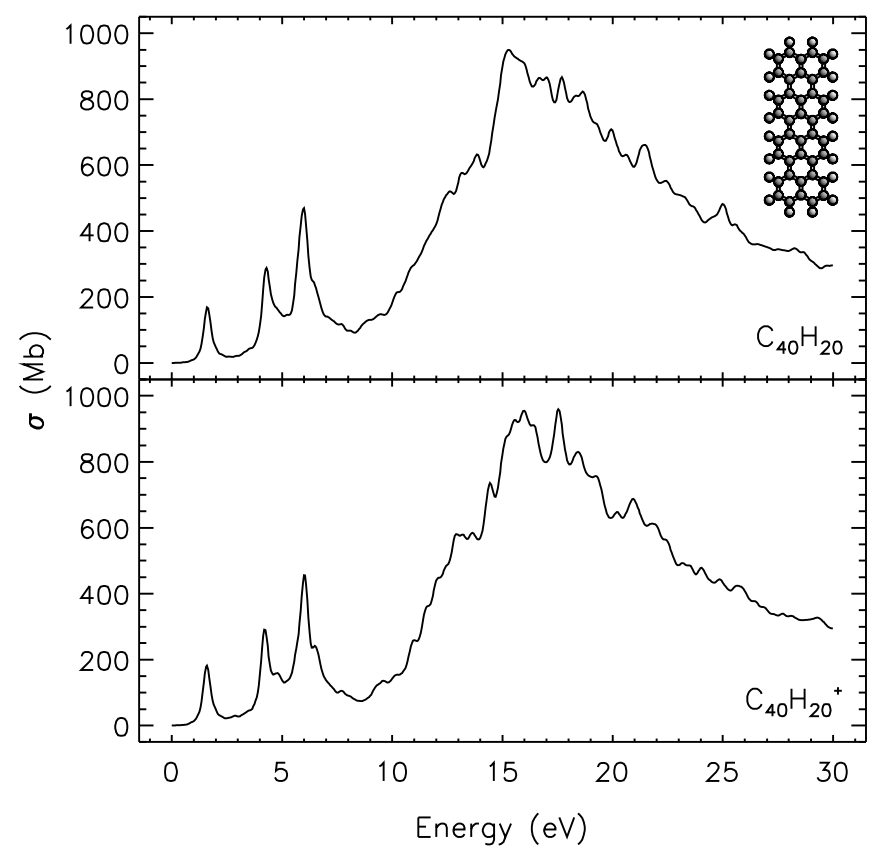

Fig. 21. Same as Fig. 3 for quaterrylene $\left(\mathrm{C}_{40} \mathrm{H}_{20}\right)$ neutral and cation.

cast some doubt on the general validity of the above assumption. Our results further show that PAH cations generally appear to display almost as strong near-UV bands as their parent neutrals, and therefore ought to be just as detectable if they were to account for AIBs. On the other hand, it is known that PAH mixtures might not exhibit sharp features in the near-UV because their bands would blend in a broad peak contributing to the well-known extinction bump at $\sim 2175 \AA$ and to the far-UV rise (Joblin 1992; Joblin et al. 1992).

To address this point, we performed a weighted sum of the single spectra presented in Figs. 3-22, assuming statistical 


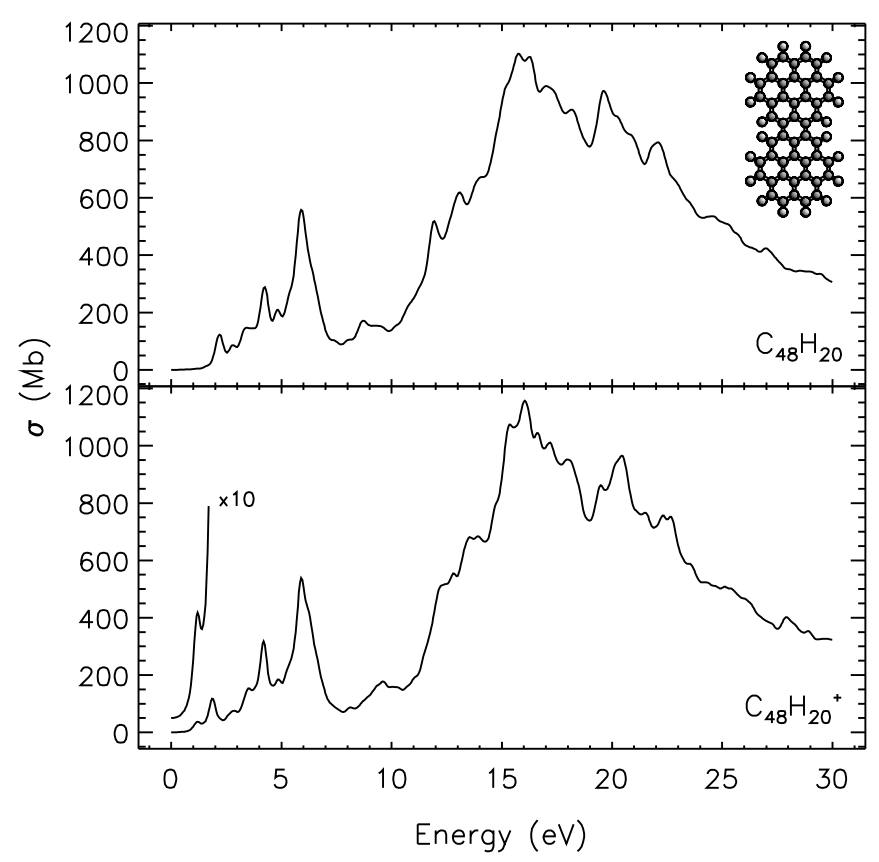

Fig. 22. Same as Fig. 3 for dicoronylene $\left(\mathrm{C}_{48} \mathrm{H}_{20}\right)$ neutral and cation.

weights to be simply inversely proportional to the total number of carbon atoms $N_{\mathrm{C}}$ of each molecule, i.e. we just made a straight average of the cross-sections normalized per carbon atom.

Figure 23 displays the overall expected spectra for our mixture of 20 different neutral and cationic PAHs. An inspection of the upper panel, corresponding to neutrals, shows that, despite the relatively small number of different species considered, together with the simplistic assumption of statistical weights of the form $1 / N_{\mathrm{C}}$, our results agree surprisingly well with the general trend observed for a natural mixture of PAHs and derivatives with $N_{\mathrm{C}}=24$ (Joblin et al. 1992). We remark that the sample of molecules we used here was not chosen to match the ones present in the experimental mixtures: our aim was to include representatives from compact and non-compact PAHs and, in addition, some molecules with five-membered aromatic rings. Our selection just happens by chance to have almost the same average number of carbon atoms per molecule as one of the mixtures measured by Joblin et al. (1992). This seems to suggest that indeed any mixture of PAHs with the same average number of carbon atoms per molecule will produce a very similar spectrum, very much regardless of the specific molecules present in it. The effect of coadding single spectra is to wash out near-UV band structures and, at the same time, to produce two distinct features:

- a collective broad absorption peak, resulting from the sum of the $\pi^{*} \leftarrow \pi$ transitions, at $\sim 6 \mathrm{eV}$, which ought to contribute to the short-wavelength side $(\sim 207 \mathrm{~nm})$ of the UV extinction bump; and

- a smooth far-UV rise.

The coadding leaves some discernible structure on the lowenergy side of the main peak, in particular two peaks which appear at $\sim 4.2$ and $\sim 4.8 \mathrm{eV}$. They are not due to a single molecule, but instead to a casual near coincidence of relatively strong bands in different molecules. We have no reason, a priori, to believe that a different sample of molecules would display similar near coincidences at the same energies, therefore we expect such structure to be more and more washed out in a larger sample, and conversely to be only visible in a sample dominated by a relatively small number of species. This might explain the negative result obtained by Clayton et al. (2003) in their search for such bands with the Hubble Space Telescope, which may be interpreted to simply mean that interstellar PAHs include a large variety of different molecules.

For the sake of completeness, we remark that in all of our synthetic spectra we assumed an intrinsic band width of $\sim 0.2 \mathrm{eV}$. This value was chosen because it is comparable with the accuracy which can be expected in TD-DFT results and because it compares favorably with available experimental data, but this theoretical method does not provide any estimate for it. If one were to assume a much smaller intrinsic bandwidth it would take a correspondingly larger number of molecules to wash out single bands from the coadded spectrum. However, as explained in detail in Sect. 2.2, electronic bands of PAHs are expected to become broader and broader for increasing energies of the excited states involved, due to their fast decay by internal conversion, hence UV bands are not expected to be very much narrower than what we assumed. Clearly, intrinsic band widths are an important parameter in deriving (upper limits of) PAH column densities from HST UV observations (Clayton et al. 2003), but they cannot be constrained with ab initio calculations at the level of theory used here. Laboratory measurements are needed to properly assess this.

There is to date no available experimental study on the overall UV spectrum of a mixture of PAH cations; however, the good agreement we obtained for neutrals between the weighted sum of our synthetic spectra and the data from Joblin et al. (1992) leads us to infer a similar validity for the weighted sum of synthetic spectra of cations. As expected, due to the similarity of their UV spectra to those of their parent neutrals, the discussion above still holds and can be transported unchanged to cations, namely:

- single features in the near-UV are lost in the mixture, UV bands blend contributing to the bump; and

- a featureless far-UV rise appears.

From the astrophysical point of view, the main lesson to be learned from Fig. 23 seems to be that if any PAHs of small to medium size, be they neutral or cationic, are to account for the far-UV rise of the interstellar extinction curve they must also contribute to the short-wavelength side of the extinction bump at $\sim 2175 \AA$. In this case, some extreme "bumpless" extinction curves, as observed e.g. by Prevot et al. (1984) towards the Small Magellanic Cloud, might be difficult to reconcile with the PAH hypothesis. Indeed, AIBs are known to be absent in the Small Magellanic Cloud.

\section{Conclusion}

Detailed information about the photo-absorption properties of neutral and ionised PAHs, which are thought to be widespread throughout ISM, will enable us to describe in a quantitative 


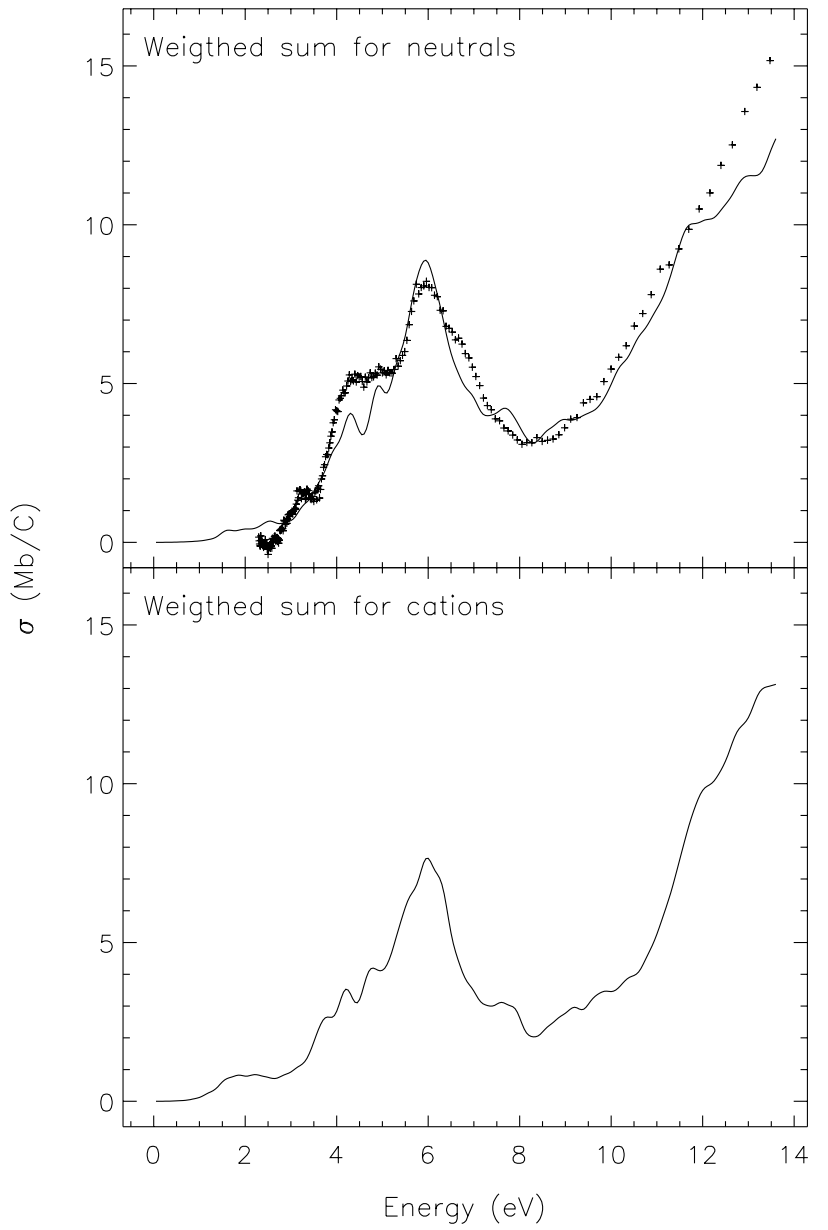

Fig. 23. Comparison between the weighted sum of the spectra displayed in Figs. 3-22, for both PAH neutrals (upper panel) and the corresponding cations (lower panel). Statistical weights are simply assumed to be proportional to the inverse of the total number of carbon atoms $N_{\mathrm{C}}$ contained in each molecule. In the upper panel we also reproduce (crosses) the experimental result obtained by Joblin et al. (1992) for a natural mixture of neutral PAHs with average $N_{\mathrm{C}}=24$ which is very close to our sample of molecules (average $N_{\mathrm{C}}=23.55$ ).

way the overall radiative balance in many interstellar environments, through the analysis of the UV to IR energy conversion.

The search for specific PAH features in space-based UV observations has recently begun (see e.g., Clayton et al. 2003), even if no such features have been detected yet. The present work suggests that indeed a large collection of different PAHs would be expected to contribute mainly to the short wavelength side of the UV bump and to the far-UV rise in the extinction curve. Additional contribution from $\pi^{*} \leftarrow \pi$ transitions in the $\mathrm{UV}$ is expected to blend into a continuum when a large number of species (more than $\sim 20$ ) is involved. This paper shows that this is the case for cations as well as for neutrals.

The good agreement between our calculated spectra of PAHs and the available experimental spectra validates their use as a quite decent substitute for laboratory data when the latter are lacking, especially for the purpose of modelling the photo-physics of specific interstellar PAHs, their interaction with the ambient radiation field, their contribution to the interstellar extinction and their resulting IR-emission, such as e.g. in Malloci et al. (2003) and Mulas et al. (2003). This will yield direct tracers for each of these species, suitable for comparison with astronomical observations e.g. by ISO and the forthcoming Herschel mission in the far-IR. In addition, this kind of approach opens the possibility to explore the above properties for other PAH-related species, such as anions, protonated and dehydrogenated PAHs, as well as possibly nitrogen and oxygen substituted species.

We emphasise, however, that the remarkable similarity we found in our theoretical UV-VUV spectra between neutral and cationic PAHs, along with the strong near-UV bands we predict for our whole sample of cations, needs to be confirmed (or refuted) by actual laboratory measurements of cations, which are very limited to date in the UV (Chillier et al. 1999) and not at all available in the VUV range.

Acknowledgements. G. Malloci gratefully acknowledges the financial support by Regione Autonoma della Sardegna which enabled a shortterm visit at CESR in Toulouse. We are thankful to the authors of octopus for their help on how to make the best use of their code. We acknowledge the High Performance Computational Chemistry Group for using their code: "NWChem, A Computational Chemistry Package for Parallel Computers, version 4.5" (2003), Pacific Northwest National Laboratory, Richland, Washington 99352-0999, USA. Part of the calculations presented here were performed using the IBM SP4 at the CINECA supercomputing facility.

\section{References}

Allamandola, L. J., Tielens, A. G. G. M., \& Barker, J. R. 1985, ApJ, 290, L25

Allamandola, L. J., Tielens, G. G. M., \& Barker, J. R. 1989, ApJS, 71, 733

Allamandola, L. J., Hudgins, D. M., \& Sandford, S. A. 1999, ApJ, 511, L115

Andriesse, C. D. 1978, A\&A, 66, 169

Bakes, E. L. O., \& Tielens, A. G. G. M. 1994, ApJ, 427, 822

Bakes, E. L. O., Tielens, A. G. G. M., \& Bauschlicher, C. W. 2001a, ApJ, 556, 501

Bakes, E. L. O., Tielens, A. G. G. M., Bauschlicher, C. W., Hudgins, D. M., \& Allamandola, L. J. 2001b, ApJ, 560, 261

Bauschlicher, C. W., \& Langhoff, S. R. 1997, Spectrochim. Acta Part A, 53, 1225

Bauschlicher, Jr., C. W. 2002, ApJ, 564, 782

Becke, A. D. 1993, J. Chem. Phys., 98, 5648

Biennier, L., Salama, F., Allamandola, L. J., \& Scherer, J. J. 2003, J. Chem. Phys., 118, 7863

Biennier, L., Salama, F., Gupta, M., \& O'Keefe, A. 2004, Chem. Phys. Lett., 387, 287

Boulanger, F. 1999, in Solid Interstellar Matter: The ISO Revolution, Les Houches Workshop, February 2-6, 1998, ed. L. d'Hendecourt, C. Joblin, \& A. Jones (EDP Sciences and Springer-Verlag), 20

Bréchignac, P., \& Pino, T. 1999, A\&A, 343, L49

Castro, A., Marques, M. A. L., Alonso, J. A., et al. 2002, J. Chem. Phys., 116, 1930

Ceperley, D. M., \& Alder, B. J. 1980, Phys. Rev. Lett., 45, 566

Chillier, X. D. F., Stone, B. M., Salama, F., \& Allamandola, L. J. 1999, J. Chem. Phys., 111, 449

Chillier, X., Boulet, P., Chermette, H., Salama, F., \& Weber, J. 2001, J. Chem. Phys., 115, 1769

Chillier, X. D. F., Stone, B. M., Joblin, C., Salama, F., \& Allamandola, L. J. 2002, J. Chem. Phys., 116, 5725 
Clayton, G. C., Gordon, K. D., Salama, F., et al. 2003, ApJ, 592, 947 Crawford, M. K., Tielens, A. G. G. M., \& Allamandola, L. J. 1985, ApJ, 293, L45

d'Hendecourt, L. B., Léger, A., Olofsson, G., \& Schmidt, W. 1986, A\&A, 170, 91

Donn, B., Allen, J., \& Khanna, R. 1989, in Interstellar Dust, IAU Symp., 135, 181

Draine, B. T. 2003, ARA\&A, 41, 241

Du, P., Salama, F., \& Loew, G. H. 1993, Chem. Phys., 173, 421

Duley, W. W., \& Williams, D. A. 1981, MNRAS, 196, 269

Ehrenfreund, P., \& Charnley, S. B. 2000, ARA\&A, 38, 427

Ehrenfreund, P., d'Hendencourt, L., Verstraete, L., et al. 1992, A\&A, 259, 257

Frish, M. J., Pople, J. A., \& Binkley, J. S. 1984, J. Chem. Phys., 80, 3265

Gudipati, M. S., Daverkausen, J., \& Hohlneicher, G. 1993, Chem. Phys., 173, 143

Gudipati, M. S., Daverkausen, J., Maus, M., \& Hohlneicher, G. 1994, Chem. Phys., 186, 289

Gudipati, M. S., Maus, M., Daverkausen, J., \& Hohlneicher, G. 1995, Chem. Phys., 192, 37

Halasinski, T. M., Hudgins, D. M., Salama, F., Allamandola, L. J., \& Bally, T. 2000, J. Phys. Chem. A, 104, 7484

Halasinski, T. M., Weisman, J., Ruiterkamp, R., et al. 2003, J. Phys. Chem. A, 107, 3660

Heinze, H. H., Görling, A., \& Rösch, N. 2000, J. Chem. Phys., 113, 2088

Hirata, S., Lee, T., \& Head-Gordon, M. 1999, J. Chem. Phys., 111, 8904

Hirata, S., Head-Gordon, M., Szczepanski, J., \& Vala, M. 2003, J. Phys. Chem. A, 107, 4940

Hudgins, D. M., Bauschlicher, C. W., \& Allamandola, L. J. 2001, Spectrochim. Acta Part A, 57, 907

Joblin, C. 1992, Ph.D. Thesis, Université Paris 7

Joblin, C., Léger, A., \& Martin, P. 1992, ApJ, 393, L79

Joblin, C., Salama, F., \& Allamandola, L. 1995, J. Chem. Phys., 102, 9743

Joblin, C., Salama, F., \& Allamandola, L. 1999, J. Chem. Phys., 110, 7287

Joblin, C., Martin, P., Malloci, G., Mulas, G., \& Léger, A. 2004, in preparation

Jochims, H. W., Baumgärtel, H., \& Leach, S. 1996, A\&A, 314, 1003

Jochims, H. W., Rühl, E., Baumgärtel, H., Tobita, S., \& Leach, S. 1997, Int. J. Mass Spectrom. \& Ion Proc., 167/168, 35

Jochims, H. W., Baumgärtel, H., \& Leach, S. 1999, ApJ, 512, 500

Kameswara-Rao, N., \& Lambert, D. L. 1993, MNRAS, 263, L27

Langhoff, S. R. 1996, J. Phys. Chem, 100, 2819

Le Page, V., Snow, T. P., \& Bierbaum, V. M. 2001, ApJS, 132, 233

Le Page, V., Snow, T. P., \& Bierbaum, V. M. 2003, ApJ, 584, 316

Leach, S. 1995, Planet. Space Sci., 43, 1153

Lee, W., \& Wdowiak, T. J. 1993, ApJ, 410, L127

Léger, A., \& d'Hendecourt, L. 1985, A\&A, 146, 81

Léger, A., d'Hendecourt, L., \& Defourneau, D. 1989, A\&A, 216, 148

Léger, A., \& Puget, J. L. 1984, A\&A, 137, L5

Li, A., \& Greenberg, J. M. 1997, A\&A, 323, 566

Malloci, G., Mulas, G., \& Benvenuti, P. 2003, A\&A, 410, 623

Marques, M. A. L., Castro, A., \& Rubio, A. 2001, J. Chem. Phys., 115,3006

Marques, M. A. L., Castro, A., Bertsch, G. F., \& Rubio, A. 2003, Computer Phys. Commun., 151, 60
Mulas, G., Malloci, G., \& Benvenuti, P. 2003, A\&A, 410, 639

Negri, F., \& Zgierski, M. Z. 1994, J. Phys. Chem., 100, 2819

Niederalt, C., Grimme, S., \& Peyerimhoff, S. D. 1995, Chem. Phys. Lett., 245, 455

Onida, G., Reining, L., \& Rubio, A. 2002, Rev. Mod. Phys., 74, 601

Ossler, F., Metz, T., \& Aldén, M. 2001a, Appl. Phys. B, 72, 465

Ossler, F., Metz, T., \& Aldén, M. 2001b, Appl. Phys. B, 72, 479

Parac, M., \& Grimme, S. 2003, Chem. Phys., 292, 11

Parisel, O., Berthier, G., \& Ellinger, Y. 1992, A\&A, 266, L1

Perdew, J. P., \& Zunger, A. 1981, Phys. Rev. B, 23, 5048

Pino, T., Boudin, N., \& Bréchignac, P. 1999, J. Chem. Phys., 111, 7337

Prevot, M. L., Lequeux, J., Prevot, L., Maurice, E., \& RoccaVolmerange, B. 1984, A\&A, 132, 389

Robinson, M. S., Beegle, L. W., \& Wdowiak, T. J. 1997, ApJ, 474, 474

Romanini, D., Biennier, L., Salama, F., et al. 1999, Chem. Phys. Lett., 303, 165

Ruiterkamp, R., Halasinski, T., Salama, F., et al. 2002, A\&A, 390, 1153

Runge, E., \& Gross, E. K. U. 1984, Phys. Rev. Lett., 52, 997

Salama, F. 1999, in Solid Interstellar Matter: The ISO Revolution, Les Houches Workshop, February 2-6, 1998, ed. L. d'Hendecourt, C. Joblin, \& A. Jones (EDP Sciences and Springer-Verlag), 65

Salama, F., \& Allamandola, L. J. 1992, ApJ, 395, 301

Salama, F., Joblin, C., \& Allamandola, L. J. 1995, Planet. Space Sci., 43,1165

Salama, F., Bakes, E. L. O., Allamandola, L. J., \& Tielens, A. G. G. M. 1996, ApJ, 458, 621

Salama, F., Galazutdinov, G. A., Krełowski, J., Allamandola, L. J., \& Musaev, F. A. 1999, ApJ, 526, 265

Scarrott, S. M., Watkin, S., Miles, J. R., \& Sarre, P. J. 1992, MNRAS, 255, 11P

Schmidt, G. D., Cohen, M., \& Margon, B. 1980, A\&A, 239, L133

Sellgren, K. 1984, ApJ, 277, 623

Stephens, P. J., Devlin, F. J., \& Chabalowski C. F., Frisch, M. 1994, J. Phys. Chem., 98, 11623

Straatsma, T. P., Apra, E., Windus, T. L., et al. 2003, NWChem, A Computational Chemistry Package for Parallel Computers, Version 4.5

Sukhorukov, O., Staicu, A., Diegel, E., et al. 2004, Chem. Phys. Lett., 386,259

Szczepanski, J., Chapo, C., \& Vala, M. 1993a, Chem. Phys. Lett., 205, 434

Szczepanski, J., Vala, M., Talbi, D., Parisel, O., \& Ellinger, Y. 1993b, J. Chem. Phys., 98, 4494

Troullier, N., \& Martins, J. L. 1991, Phys. Rev. B, 43, 1993

Vala, M., Szczepanski, J., Pauzat, O., et al. 1994, J. Phys. Chem., 98, 9187

van der Zwet, G. P., \& Allamandola, L. 1985, A\&A, 146, 76

Van Winckel, H., Cohen, M., \& Gull, T. R. 2002, A\&A, 390, 147

Verstraete, L., \& Léger, A. 1992, A\&A, 266, 513

Verstraete, L., Léger, A., d'Hendecourt, L., Dutuit, O., \& Défourneau, D. 1990, A\&A, 237, 436

Weisman, J. L., Lee, T. J., \& Head-Gordon, M. 2001, Spectrochim. Acta Part A, 57, 931

Weisman, J. L., Lee, T. J., Salama, F., \& Head-Gordon, M. 2003, ApJ, 587,256

Yabana, K., \& Bertsch, G. F. 1999, Int. J. Q. Chem., 75, 55 